\title{
Calculating differential Galois groups of parametrized differential equations, with applications to hypertranscendence
}

\author{
Charlotte Hardouin · Andrei Minchenko · \\ Alexey Ovchinnikov
}

Received: 8 June 2015 / Accepted: 16 June 2016

\begin{abstract}
The main motivation of our work is to create an efficient algorithm that decides hypertranscendence of solutions of linear differential equations, via the parameterized differential and Galois theories. To achieve this, we expand the representation theory of linear differential algebraic groups and develop new algorithms that calculate unipotent radicals of parameterized differential Galois groups for differential equations whose coefficients are rational functions. P. Berman and M.F. Singer presented an algorithm calculating the differential Galois group for differential equations without parameters whose differential operator is a composition of two completely reducible differential operators. We use their algorithm as a part of our algorithm. As a result, we find an effective criterion for the algebraic independence of the solutions of parameterized differential equations and all of their derivatives with respect to the parameter.
\end{abstract}

Keywords Differential Galois theory $\cdot$ Differential transcendence $\cdot$ Special functions

This work was partially supported by by ANR-11-LABX-0040-CIMI within the program ANR-11-IDEX0002-02, by ANR-10-JCJC 0105, by the NSF grants CCF-0952591 and DMS-1413859, by the NSA grant H98230-15-1-0245, by the ISF grant 756/12, and by the Minerva foundation with funding from the Federal German Ministry for Education and Research.

C. Hardouin

Institut de Mathématiques de Toulouse, 118 route de Narbonne, 31062 Toulouse Cedex 9, France

E-mail: hardouin@math.ups-tlse.fr

A. Minchenko

The Weizmann Institute of Science, Faculty of Mathematics and Computer Science, 234 Herzl Street, Rehovot, 7610001, Israel

E-mail: an.minchenko@gmail.com

A. Ovchinnikov

CUNY Queens College, Department of Mathematics, 65-30 Kissena Blvd, Queens, NY 11367, USA and CUNY Graduate Center, Department of Mathematics, 365 Fifth Avenue, New York, NY 10016, USA

E-mail: aovchinnikov@qc.cuny.edu 


\section{Contents}

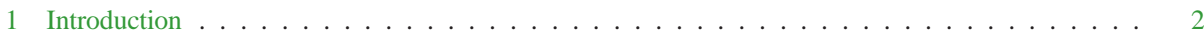

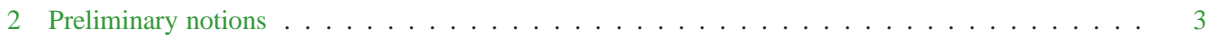

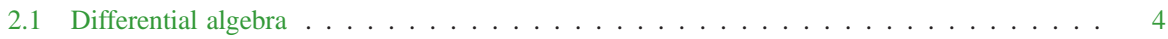

2.2 Linear differential algebraic groups and their unipotent radicals . . . . . . . . . . . . 4

2.3 Parameterized differential modules . . . . . . . . . . . . . . . . . 10

3 Calculating the parameterized differential Galois group of $L_{1}\left(L_{2}(y)\right)=0 \ldots \ldots \ldots \ldots \ldots$

3.1 Structure of the parameterized differential Galois group $\ldots \ldots \ldots \ldots \ldots$

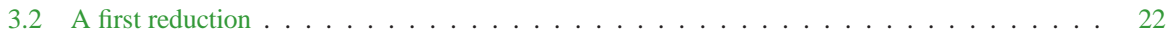

3.3 The unipotent radical of the parameterized differential Galois group of an extension . . . . 25

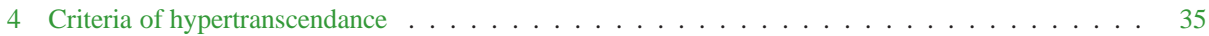

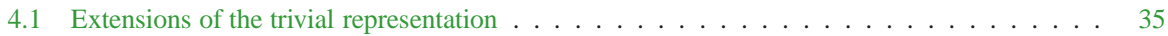

4.2 A practical criterion of hypertranscendance . . . . . . . . . . . . . . . . 39

4.3 Application to the Lommel equation . . . . . . . . . . . . . . . . . . . . . 40

\section{Introduction}

A special function is said to be hypertranscendental if it does not satisfy any algebraic differential equation. The study of functional hypertranscendence has recently appeared in various areas of mathematics. In combinatorics, the question of the hypertranscendence of generating series is frequent because it gives information on the growth of the coefficients: for instance, the work of Kurkova and Raschel [30] solved a famous conjecture about the differential algebraic behaviour of generating series of walks on the plane. Dreyfus, Roques, and Hardouin [18] gave criteria to test the hypertranscendence of generating series associated to $p$-automatic sequences and more generally Mahler functions, generalizing the work of Nguyen [40], Nishioka [41], and Randé [46]. Also, when the derivation encodes the continuous deformation of an auxiliary parameter, the hypertranscendence is connected to the notion of isomonodromic deformation (see the work of Mitschi and Singer [37]).

The work of Cassidy, Hardouin, and Singer [13,22] were motivated by a study of hypertranscendence using Galois theory. Starting from a linear functional equation with coefficients in a field with a "parametric" derivation, they were able to construct a geometric object, called the parameterized differential Galois group, whose symmetries control the algebraic relations between the solutions of the functional equation and all of their derivatives. The question of hypertranscendence of solutions of linear functional equations is thus reduced to the computation of the parameterized differential Galois groups of the equations (see for instance the work of Arreche [1] on the incomplete gamma function $\gamma(x, t)$ and the work [18]). The parameterized differential Galois groups are linear differential algebraic groups as introduced by Kolchin and developed by Cassidy [8]. These are groups of matrices whose entries satisfy systems of polynomial differential equations, called defining equations of the parameterized differential Galois group.

Then, in this context of Galois theory, one can address a direct problem, that is, the question of the algorithmic computation of the parameterized differential Galois group. 
For linear functional equations of order 2, one can find a Kovacic-type algorithm initiated by Dreyfus [17] and completed by Arreche [2]. In [36], Minchenko, Ovchinnikov, and Singer gave an algorithm that allows to test if the parameterized differential Galois group is reductive and to compute the group in that case. In [35], they also show how to compute the parameterized differential Galois group if its quotient by the unipotent radical is conjugate to a group of matrices with constant entries with respect to the parametric derivations. The algorithms of [35,36] rely on bounds on the order of the defining equations of the parameterized differential Galois group, which allows to use the algorithm obtained by Hrushovski [24] and has been further analyzed and improved by Feng [19] in the case of no parametric derivations.

In this paper, we study the parameterized differential Galois group of a differential operator of the form $L_{1}\left(L_{2}(y)\right)=0$ where $L_{1}, L_{2}$ are completely reducible differential operators. This situation goes beyond the previously studied cases, because the parameterized Galois group of such an equation is no longer reductive and its quotient by its unipotent radical might not be constant. If there is no parametric derivation, this problem was solved by Berman and Singer in [4] for differential operators and rephrased using Tannakian categories by Hardouin [21]. The general case is however more complicated because, unlike the case of no parameters, the order of the defining equations of the parameterized differential Galois group is no longer controlled by the order of the functional equation $L_{1}\left(L_{2}(y)\right)=0$. Therefore, we present an algorithm that relies on bounds (see Section 3.3.3) and, in a generic situation, we find a description of the parameterized differential Galois group. In this description, the defining equations of the unipotent radical are obtained by applying standard operations to linear differential operators (cf. [21]).

However, by a careful study of the extension of completely reducible representations of quasi-simple linear differential algebraic groups, we are able to deduce a complete and effective criterion to test the hypertranscendence of solutions of inhomogeneous linear differential equations (Theorem 4.7).

The paper is organized as follows. We start with a brief review of the basic notions in differential algebra, linear differential algebraic groups, and linear differential equations with parameters in Section 2. Our algorithmic results for calculating parameterized differential Galois groups are presented in Section 3. Our effective criterion for hypertranscendence of solutions of extensions of irreducible differential equations is contained in Section 4.2, which is preceded by Section 4.1, where we extend results of Minchenko and Ovchinnikov [34] for the purposes of the hypertranscendence criterion. We use this criterion to prove hypertranscendence results for the Lommel differential equation in Section 4.3.

\section{Preliminary notions}

We shall start with some basic notions of differential algebra and then recall what linear differential algebraic groups and their representations are. 


\subsection{Differential algebra}

Definition 2.1 A differential ring is a ring $R$ with a finite set $\Delta=\left\{\delta_{1}, \ldots, \delta_{m}\right\}$ of commuting derivations on $R$. A $\Delta$-ideal of $R$ is an ideal of $R$ stable under any derivation in $\Delta$.

In the present paper, $\Delta$ will consist of one or two elements. Let $R$ be a $\Delta$-ring. For any $\delta \in \Delta$, we denote

$$
R^{\delta}=\{r \in R \mid \delta(r)=0\},
$$

which is a $\Delta$-subring of $R$ and is called the ring of $\delta$-constants of $R$. If $R$ is a field and a differential ring, then it is called a differential field, or $\Delta$-field for short. For example, $R=\mathbb{Q}(x, t), \Delta=\{\delta, \partial\}$, and $\partial=\partial / \partial x, \delta=\partial / \partial t$, forms a differential field. The notion of $R$ - $\Delta$-algebra is defined analogously.

The ring of $\Delta$-differential polynomials $K\left\{y_{1}, \ldots, y_{n}\right\}$ in the differential indeterminates, or $\Delta$-indeterminates, $y_{1}, \ldots, y_{n}$ and with coefficients in a $\Delta$-field $(K, \Delta)$, is the ring of polynomials in the indeterminates formally denoted

$$
\left\{\delta_{1}^{i_{1}} \cdot \ldots \cdot \delta_{m}^{i_{m}} y_{i} \mid i_{1}, \ldots, i_{m} \geq 0,1 \leq i \leq n\right\}
$$

with coefficients in $K$. We endow this ring with a structure of $K$ - $\Delta$-algebra by setting

$$
\delta_{k}\left(\delta_{1}^{i_{1}} \cdot \ldots \cdot \delta_{m}^{i_{m}} y_{i}\right)=\delta_{1}^{i_{1}} \cdot \ldots \cdot \delta_{k}^{i_{k}+1} \cdot \ldots \cdot \delta_{m}^{i_{m}} y_{i}
$$

Definition 2.2 (see [32, Corollary 1.2(ii)]) A differential field $(K, \Delta)$ is said to be differentially closed or $\Delta$-closed for short, if, for every (finite) set of $\Delta$-polynomials $F \subset K\left\{y_{1}, \ldots, y_{n}\right\}$, if the system of differential equations $F=0$ has a solution with entries in some $\Delta$-field extension $L$, then it has a solution with entries in $K$.

For $\partial \in \Delta$, the ring $K[\partial]$ of differential operators, or $\partial$-operators for short, is the $K$-vector space with basis $1, \partial, \ldots, \partial^{n}, \ldots$ endowed with the following multiplication rule:

$$
\partial \cdot a=a \cdot \partial+\partial(a) .
$$

To a $\partial$-operator $L$ as above, one can associate the linear homogeneous $\partial$-polynomial

$$
L(y)=a_{n} \partial^{n} y+\ldots+a_{1} \partial y+a_{0} y \in K\{y\} .
$$

In what follows, we assume that every field is of characteristic zero.

\subsection{Linear differential algebraic groups and their unipotent radicals}

In this section, we first introduce the basic terminology of Kolchin-closed sets, linear differential algebraic groups and their representations. We then define unipotent radicals of linear differential algebraic groups, reductive linear differential algebraic groups and their structural properties. We continue with the notion of conjugation to constants of linear differential algebraic groups.

Let $(\mathbf{k}, \delta)$ be a differentially closed field, $C=\mathbf{k}^{\delta}$, and $(F, \delta)$ a $\delta$-subfield of $\mathbf{k}$. 


\subsubsection{First definitions}

Definition 2.3 A Kolchin-closed (or $\delta$-closed, for short) set $W \subset \mathbf{k}^{n}$ is the set of common zeroes of a system of $\delta$-polynomials with coefficients in $\mathbf{k}$, that is, there exists $S \subset \mathbf{k}\left\{y_{1}, \ldots, y_{n}\right\}$ such that

$$
W=\left\{a \in \mathbf{k}^{n} \mid f(a)=0 \text { for all } f \in S\right\} .
$$

We say that $W$ is defined over $F$ if $W$ is the set of zeroes of $\delta$-polynomials with coefficients in $F$. More generally, for an $F$ - $\delta$-algebra R,

$$
W(R)=\left\{a \in R^{n} \mid f(a)=0 \text { for all } f \in S\right\} .
$$

Definition 2.4 If $W \subset \mathbf{k}^{n}$ is a Kolchin-closed set defined over $F$, the $\delta$-ideal

$$
\mathbb{I}(W)=\left\{f \in F\left\{y_{1}, \ldots, y_{n}\right\} \mid f(w)=0 \text { for all } w \in W(\mathbf{k})\right\}
$$

is called the defining $\delta$-ideal of $W$ over $F$. Conversely, for a subset $S$ of $F\left\{y_{1}, \ldots, y_{n}\right\}$, the following subset is $\delta$-closed in $\mathbf{k}^{n}$ and defined over $F$ :

$$
\mathbf{V}(S)=\left\{a \in \mathbf{k}^{n} \mid f(a)=0 \text { for all } f \in S\right\} .
$$

Remark 2.5 Since every radical $\delta$-ideal of $F\left\{y_{1}, \ldots, y_{n}\right\}$ is generated as a radical $\delta$ ideal by a finite set of $\delta$-polynomials (see, for example, [47, Theorem, page 10], [27, Sections VII.27-28]), the Kolchin topology is Ritt-Noetherian, that is, every strictly decreasing chain of Kolchin-closed sets has a finite length.

Definition 2.6 Let $W \subset \mathbf{k}^{n}$ be a $\delta$-closed set defined over $F$. The $\delta$-coordinate ring $F\{W\}$ of $W$ over $F$ is the $F$ - $\Delta$-algebra

$$
F\{W\}=F\left\{y_{1}, \ldots, y_{n}\right\} / \mathbb{I}(W) .
$$

If $F\{W\}$ is an integral domain, then $W$ is said to be irreducible. This is equivalent to $\mathbb{I}(W)$ being a prime $\delta$-ideal.

Example 2.7 The affine space $\mathbf{A}^{n}$ is the irreducible Kolchin-closed set $\mathbf{k}^{n}$. It is defined over $F$, and its $\delta$-coordinate ring over $F$ is $F\left\{y_{1}, \ldots, y_{n}\right\}$.

Definition 2.8 Let $W \subset \mathbf{k}^{n}$ be a $\delta$-closed set defined over $F$. Let $\mathbb{I}(W)=\mathfrak{p}_{1} \cap \ldots \cap$ $\mathfrak{p}_{q}$ be a minimal $\delta$-prime decomposition of $\mathbb{I}(W)$, that is, the $\mathfrak{p}_{i} \subset F\left\{y_{1}, \ldots, y_{n}\right\}$ are prime $\delta$-ideals containing $\mathbb{I}(W)$ and minimal with this property. This decomposition is unique up to permutation (see [27, Section VII.29]). The irreducible Kolchin-closed sets $W_{i}=\mathbf{V}\left(\mathfrak{p}_{i}\right)$ are defined over $F$ and called the irreducible components of $W$. We have $W=W_{1} \cup \ldots \cup W_{q}$.

Definition 2.9 Let $W_{1} \subset \mathbf{k}^{n_{1}}$ and $W_{2} \subset \mathbf{k}^{n_{2}}$ be two Kolchin-closed sets defined over $F$. A $\delta$-polynomial map (morphism) defined over $F$ is a map

$$
\varphi: W_{1} \rightarrow W_{2}, \quad a \mapsto\left(f_{1}(a), \ldots, f_{n_{2}}(a)\right), a \in W_{1},
$$

where $f_{i} \in F\left\{y_{1}, \ldots, y_{n_{1}}\right\}$ for all $i=1, \ldots, n_{2}$.

If $W_{1} \subset W_{2}$, the inclusion map of $W_{1}$ in $W_{2}$ is a $\delta$-polynomial map. In this case, we say that $W_{1}$ is a $\delta$-closed subset of $W_{2}$. 
Example 2.10 Let $\mathrm{GL}_{n} \subset \mathbf{k}^{n}$ be the group of $n \times n$ invertible matrices with entries in k. One can see $\mathrm{GL}_{n}$ as a Kolchin-closed subset of $\mathbf{k}^{n^{2}} \times \mathbf{k}$ defined over $F$, defined by the equation $\operatorname{det}(X) y-1$ in $F\left\{\mathbf{k}^{n^{2}} \times \mathbf{k}\right\}=F\{X, y\}$, where $X$ is an $n \times n$-matrix of $\delta$-indeterminates over $F$ and $y$ a $\delta$-indeterminate over $F$. One can thus identify the $\delta$ coordinate ring of $\mathrm{GL}_{n}$ over $F$ with $F\{Y, 1 / \operatorname{det}(Y)\}$, where $Y=\left(y_{i, j}\right)_{1 \leq i, j \leq n}$ is a matrix of $\delta$-indeterminates over $F$. We also denote the special linear group that consists of the matrices of determinant 1 by $\mathrm{SL}_{n} \subset \mathrm{GL}_{n}$.

Similarly, if $V$ is a finite-dimensional $F$-vector space, $\operatorname{GL}(V)$ is defined as the group of invertible k-linear maps of $V \otimes_{F} \mathbf{k}$. To simplify the terminology, we will also treat $\mathrm{GL}(V)$ as Kolchin-closed sets tacitly assuming that some basis of $V$ over $F$ is fixed.

Remark 2.11 If $K$ is a field, we denote the group of invertible matrices with coefficients in $K$ by $\mathrm{GL}_{n}(K)$.

Definition 2.12 ([8, Chapter II, Section 1, page 905]) A linear differential algebraic group $G \subset \mathbf{k}^{n^{2}}$ defined over $F$ is a subgroup of $\mathrm{GL}_{n}$ that is a Kolchin-closed set defined over $F$. If $G \subset H \subset \mathrm{GL}_{n}$ are Kolchin-closed subgroups of $\mathrm{GL}_{n}$, we say that $G$ is a $\delta$-closed subgroup, or $\delta$-subgroup of $H$.

Proposition 2.13 Let $G \subset \mathrm{GL}_{n}$ be a linear algebraic group defined over $F$. We have:

(1) $G$ is a linear differential algebraic group.

(2) Let $H \subset G$ be a $\delta$-subgroup of $G$ defined over $F$, and the Zariski closure $\bar{H} \subset G$ be the closure of $H$ with respect to the Zariski topology. In this case, $\bar{H}$ is a linear algebraic group defined over $F$, whose polynomial defining ideal over $F$ is

$$
\mathbb{I}(H) \cap F[Y] \subset \mathbb{I}(H) \subset F\{Y\}
$$

where $Y=\left(y_{i, j}\right)_{1 \leq i, j \leq n}$ is a matrix of $\delta$-indeterminates over $F$.

Definition 2.14 Let $G$ be a linear differential algebraic group defined over $F$. The irreducible component of $G$ containing the identity element $e$ is called the identity compo$n e n t$ of $G$ and denoted by $G^{\circ}$. The linear differential algebraic group $G^{\circ}$ is a $\delta$-subgroup of $G$ defined over $F$. The linear differential algebraic group $G$ is said to be connected if $G=G^{\circ}$, which is equivalent to $G$ being an irreducible Kolchin-closed set [8, page 906].

Definition 2.15 ([9],[43, Definition 6]) Let $G$ be a linear differential algebraic group defined over $F$ and let $V$ be a finite-dimensional vector space over $F$. A $\delta$-polynomial group homomorphism $\rho: G \rightarrow \mathrm{GL}(V)$ defined over $F$ is called a representation of $G$ over $F$. We shall also say that $V$ is a $G$-module over $F$. By a faithful (respectively, simple, semisimple) $G$-module, we mean a faithful (respectively, irreducible, completely reducible) representation $\rho: G \rightarrow \mathrm{GL}(V)$.

The image of a $\delta$-polynomial group homomorphism $\rho: G \rightarrow H$ is Kolchin closed [8, Proposition 7]. Moreover, if $\operatorname{ker}(\rho)=\{e\}$, then $\rho$ is an isomorphism of linear differential algebraic groups between $G$ and $\rho(G)$ [8, Proposition 8]. 
Definition 2.16 ([10, Theorem 2]) A linear differential algebraic group $G$ is unipotent if one of the following equivalent conditions holds:

(1) $G$ is conjugate to a differential algebraic subgroup of the group of unipotent upper triangular matrices;

(2) $G$ contains no elements of finite order $>1$;

(3) $G$ has a descending normal sequence of differential algebraic subgroups

$$
G=G_{0} \supset G_{1} \supset \ldots \supset G_{N}=\{e\}
$$

with $G_{i} / G_{i+1}$ isomorphic to a differential algebraic subgroup of the additive group $\mathbf{G}_{a}$.

One can show that a linear differential algebraic group $G$ defined over $F$ admits a largest normal unipotent differential algebraic subgroup defined over $F$ [33, Theorem 3.10].

Definition 2.17 Let $G$ be a linear differential algebraic group defined over $F$. The largest normal unipotent differential algebraic subgroup of $G$ defined over $F$ is called the unipotent radical of $G$ and denoted by $R_{u}(G)$. The unipotent radical of a linear algebraic group $H$ is also denoted by $R_{u}(H)$.

Note that, for a linear differential algebraic group $G$, we always have

$$
\overline{R_{u}(G)} \subset R_{u}(\bar{G})
$$

and this inclusion can be strict [33, Example 3.17].

\subsubsection{Almost direct products and reductive linear differential algebraic group}

We recall what reductive linear differential algebraic groups are and how they decompose into almost direct products of tori and quasi-simple subgroups.

Definition 2.18 A linear differential algebraic group $G$ is said to be simple if $\{e\}$ and $G$ are the only normal differential algebraic subgroups of $G$.

Definition 2.19 A quasi-simple linear (differential) algebraic group is a finite central extension of a simple non-commutative linear (differential) algebraic group.

Definition 2.20 ([33, Definition 3.12]) A linear differential algebraic group $G$ defined over $F$ is said to be reductive if $R_{u}(G)=\{e\}$.

By definition, the following holds for linear differential algebraic groups:

$$
\text { simple } \Longrightarrow \text { quasi-simple } \Longrightarrow \text { reductive. }
$$

Example 2.21 $\mathrm{SL}_{2}$ is quasi-simple but not simple, while $\mathrm{PSL}_{2}$ is simple. 
Proposition 2.22 ([36, Remark 2.9]) Let $G \subset \mathrm{GL}_{n}$ be a linear differential algebraic group defined over $F$. If $\bar{G} \subset \mathrm{GL}_{n}$ is a reductive linear algebraic group, then $G$ is a reductive linear differential algebraic group.

Proposition 2.23 Let $G \subset \mathrm{GL}(V)$ be a linear differential algebraic group. The following statements are equivalent:

(1) the G-module $V$ is semisimple;

(2) $V$ is semisimple as a $\bar{G}$-module, where $\bar{G} \subset \mathrm{GL}(V)$ stands for the Zariski closure;

(3) $\bar{G}$ is reductive;

(4) $V$ is semisimple as a $\bar{G}^{\circ}$-module;

(5) $V$ is semisimple as $a G^{\circ}$-module.

Proof For every subspace $U \subset V$, the set $N$ of elements $g \in \mathrm{GL}(V)$ preserving $U$ is an algebraic subgroup of $\mathrm{GL}(V)$. Therefore, $U$ is $G$-invariant if and only if it is $\bar{G}$-invariant:

$$
G \subset N \Leftrightarrow \bar{G} \subset N .
$$

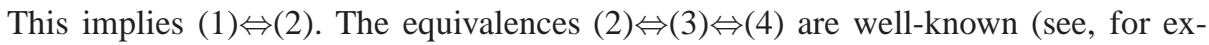
ample, [50, Chapter 2]). Since the Kolchin topology contains the Zariski topology of $\mathrm{GL}(V), \overline{G^{\circ}}$ is Zariski irreducible, hence, equals $\bar{G}^{\circ}$. Applying $(1) \Leftrightarrow(2)$ to the case of a connected $G$, we obtain $(4) \Leftrightarrow(5)$.

Definition 2.24 Let $G$ be a group and $G_{1}, \ldots, G_{n}$ some subgroups of $G$. We say that $G$ is the almost direct product of $G_{1}, \ldots, G_{n}$ if

(1) the commutator subgroups $\left[G_{i}, G_{j}\right]=\{e\}$ for all $i \neq j$;

(2) the morphism

$$
\psi: G_{1} \times \ldots \times G_{n} \rightarrow G, \quad\left(g_{1}, \ldots, g_{n}\right) \mapsto g_{1} \cdot \ldots \cdot g_{n}
$$

is an isogeny, that is, a surjective map with a finite kernel.

We summarize some results on the decomposition of reductive, algebraic and differential algebraic, groups in the theorem below. We refer to Definition 2.3 for the notation $G(C)$ with $G$ a linear (differential) algebraic group defined over $C$.

Theorem 2.25 Let $G \subset \mathrm{GL}_{n}$ be a linear differential algebraic group defined over $F$. Assume that $\bar{G} \subset \mathrm{GL}_{n}$ is a connected reductive algebraic group. Then

(1) $\bar{G}$ is an almost direct product of a torus $H_{0}$ and non-commutative normal quasisimple linear algebraic groups $H_{1}, \ldots, H_{s}$ defined over $\mathbb{Q}$;

(2) $G$ is an almost direct product of a Zariski dense $\delta$-closed subgroup $G_{0}$ of $H_{0}$ and some $\delta$-closed subgroups $G_{i}$ of $H_{i}$ for $i=1, \ldots, s$;

(3) moreover, either $G_{i}=H_{i}$ or $G_{i}$ is conjugate by a matrix of $H_{i}$ to $H_{i}(C)$;

The $H_{i}$ 's are called the quasi-simple components of $\bar{G}$; the $G_{i}$ 's are called the $\delta$-quasisimple components of $G$. 
Proof Part (1) can be found in [25, Theorem 27.5, page 167]. Parts (2) and (3) are contained in [33, proof of Lemma 4.5] and [11, Theorems 15 and 18].

Remark 2.26 As noticed in [36, Section 5.3.1], the decomposition of $\bar{G}$ as above can be made effective.

Proposition 2.27 If $v: G_{1} \times G_{2} \rightarrow G$ is a surjective homomorphism of linear differential algebraic groups and $V$ is a simple $G$-module, then $V$, viewed as a $G_{1} \times G_{2}$-module via $v$, is isomorphic to $V_{1} \otimes V_{2}$, where each $V_{i}$ is a simple $G_{i}$-module.

Proof Since $v$ is surjective, $V$ is simple as a $G_{1} \times G_{2}$-module. Let $V_{1}$ be a simple (nonzero) $G_{1}$-submodule of $V$ and $U \subset V$ the sum of all $G_{1}$-submodules isomorphic to $V_{1}$. Since all elements of $G_{2}$ send $V_{1}$ to an isomorphic submodule, we obtain that $U$ is $G_{1} \times$ $G_{2}$-invariant. Since $V$ is $G_{1} \times G_{2}$-simple, $U=V$. We choose a direct sum decomposition

$$
V=\bigoplus_{j \in J} U_{j}, \quad U_{j} \cong V_{1} \text { for all } j \in J
$$

and, for each $j \in J$, a non-zero $u_{j} \in U_{j}$, and let $V_{2}=\operatorname{span}_{j \in J}\left\{u_{j}\right\} \subset V$. We see that, as $G_{1}$-modules, $V \cong V_{1} \otimes V_{2}$, where $G_{1}$ acts trivially on $V_{2}$.

By [51, Exercise 11.30], every endomorphism of $V_{1} \otimes V_{2}$ commuting with the action of $G_{1}$ has the form $\mathrm{id}_{V_{1}} \otimes A$, where $A$ is an endomorphism of $V_{2}$. This means that $V_{2}$ has a structure of a $G_{2}$-module such that the $G_{1}$-module isomorphism $V \cong V_{1} \otimes V_{2}$ extends to a $G_{1} \times G_{2}$-module isomorphism. Since $V$ is $G_{1} \times G_{2}$-simple, $V_{2}$ is $G_{2}$-simple. It remains to note that the representation $G_{i} \rightarrow \mathrm{GL}\left(V_{i}\right), i=1,2$, is differential since it is isomorphic to a subrepresentation of the representation $G_{i} \rightarrow \mathrm{GL}(V)$.

Definition 2.28 A connected linear differential algebraic group $T$ is called a $\delta$-torus if there is an isomorphism $\alpha$ of $T$ onto a Zariski dense $\delta$-subgroup $T^{\prime} \subset\left(\mathbf{k}^{\times}\right)^{n}, n \geq 0$.

Let $T_{C}^{\prime}=\left(C^{\times}\right)^{n}$. By [8, Proposition 31], $T_{C}^{\prime} \subset T^{\prime}$. Let $T_{C}=\alpha^{-1}\left(T_{C}^{\prime}\right)$. The $\delta$-subgroup $T_{C}$ does not depend on the choice of $\alpha$ : since any differential homomorphism $\left(C^{\times}\right)^{n} \rightarrow$ $\left(\mathbf{k}^{\times}\right)^{m}$ is monomial in each of the $m$ components, its image is contained in $\left(C^{\times}\right)^{m}$.

Corollary 2.29 Let $G \subset \mathrm{GL}(V)$ be a connected linear differential algebraic group. If the $G$-module $V$ is simple and non-constant, then there exists a $\delta$-torus $T \subset G$ such that $V$ is semisimple and non-constant as a $T$-module.

Proof Since $V$ is simple, $G$ is reductive by Proposition 2.23. By Theorem 2.25, $G$ decomposes as an almost direct product of a $\delta$-torus $G_{0}$ and $\delta$-quasi-simple components $G_{i}, 1 \leq i \leq s$. By Proposition 2.27, $V$ is a tensor product of simple $G_{i}$-modules $W_{i}$. By [33, Theorem 3.3], representations of $G_{i}$ on $W_{i}$ are polynomial, that is, extend to algebraic representations $\rho_{i}: \overline{G_{i}} \rightarrow \mathrm{GL}\left(W_{i}\right)$.

Since $V$ is non-constant, there is an $i, 0 \leq i \leq s$, such that $W_{i}$ is non-constant. If $i>0$, then $G_{i}=\overline{G_{i}}$. Indeed, otherwise $G_{i} \simeq H(C)$, where $H=\overline{G_{i}}$ is a quasi-simple algebraic group defined over $C$ (see Theorem 2.25). Since all algebraic representations of $H$ are defined over $\mathbb{Q}$ (see, for example, [5, Section 5]), $\rho_{i}\left(G_{i}\right)$ is conjugate to constants, which contradicts the assumption on $W_{i}$. Thus, $G_{i}=\overline{G_{i}}$, and we can take $T$ to be a maximal torus of $G_{i}$ (see [25, Sections 21.3-21.4]). If $i=0$, let $T=G_{0}$. 


\subsubsection{Conjugation to constants}

Conjugation to constants will play an essential role in our arguments. We recall what it means. As before, $\mathbf{k}$ is a differentially closed field containing $F$ and $C$ is the field of $\delta$-constants of $\mathbf{k}$.

Definition 2.30 Let $G \subset \mathrm{GL}_{n}$ be a linear algebraic group over $F$. We say that $G$ is conjugate to constants if there exists $h \in \mathrm{GL}_{n}$ such that $h G h^{-1} \subset \mathrm{GL}_{n}(C)$. Similarly, we say that a representation $\rho: G \rightarrow \mathrm{GL}_{n}$ is conjugate to constants if $\rho(G)$ is conjugate to constants in $\mathrm{GL}_{n}$.

Proposition 2.31 Let $\rho: G \subset \mathrm{GL}(W) \rightarrow \mathrm{GL}(V)$ be a representation of a linear differential algebraic group $G$ such that $\bar{G} \subset \mathrm{GL}(W)$ is a connected reductive linear algebraic group. Assume that $\rho$ is defined over the field $C$. With notation of Theorem 2.25, assume that $Z$ acts by constant weights on $V$ and that, for all $i=1, \ldots, s$, either $H_{i} \neq G_{i}$ or $\left.\rho\right|_{H_{i}}$ is the identity. Then there exists $g \in \bar{G}$ such that

$$
\rho\left(g G g^{-1}\right) \subset \mathrm{GL}(V)(C) .
$$

Proof Let $S=\left\{i \mid H_{i}=G_{i}\right\}$. By assumption, $\rho\left(H_{i}\right)=\{1\}$ for all $i \in S$. By Theorem 2.25, for all $i \notin S$, there exists $g_{i} \in G_{i}$ such that $g_{i} H_{i} g_{i}^{-1} \subset G_{i}(C)$. Set

$$
g=\prod_{i \in S} g_{i} \in G
$$

Let $h \in G$. Since $G$ is the almost direct product of $Z$ and of its $\delta$-quasi-simple components, there exist $z \in Z$ and, for $i \in\{1, \ldots, s\}$, an element $h_{i} \in H_{i}$ such that $h=z h_{1} \cdot \ldots \cdot h_{s}$. Now,

$$
\rho\left(g h g^{-1}\right)=\rho(z) \prod_{i \notin S} \rho\left(g_{i} h_{i} g_{i}^{-1}\right) .
$$

Since $\rho$ is defined over the constants and $g_{i} h_{i} g_{i}^{-1} \in G_{i}(C)$ for all $i \notin S$, we find that

$$
\rho\left(g_{i} h_{i} g_{i}^{-1}\right) \subset \mathrm{GL}(V)(C) .
$$

Since $\rho(z)$ is also constant, the same holds for $\rho\left(g h g^{-1}\right)$.

\subsection{Parameterized differential modules}

In this section, we recall the basic definitions of differential modules and prolongation functors for differential modules with parameters. We then continue with the notion of complete integrability of differential modules and its relation to conjugation to constants of parameterized differential Galois groups. We also show a new result, Proposition 2.54, which relates the conjugation to constants of a linear differential algebraic group and of its identity component. 


\subsubsection{Differential modules and prolongations}

Let $K$ be a $\Delta=\{\partial, \delta\}$-field. We denote by $\mathbf{k}$ (respectively, $C$ ) the field of $\partial$ (respectively, $\Delta$ )-constants of $K$. We assume for simplicity that $(\mathbf{k}, \delta)$ is differentially closed (this assumption was relaxed in $[20,53,39])$. Therefore, unless explicitly mentioned, any Kolchin-closed set considered in the rest of the paper is a subset of some $\mathbf{k}^{n}$.

Definition 2.32 A $\partial$-module $\mathscr{M}$ over $K$ is a left $K[\partial]$-module that is a finitedimensional vector space over $K$.

Let $\mathscr{M}$ be a $\partial$-module over $K$ and let $\left\{e_{1}, \ldots, e_{n}\right\}$ be a $K$-basis of $\mathscr{M}$. Let $A=$ $\left(a_{i, j}\right) \in K^{n \times n}$ be the matrix defined by

$$
\partial\left(e_{i}\right)=-\sum_{j=1}^{n} a_{j, i} e_{j}, \quad i=1, \ldots, n .
$$

Then, for any element $m=\sum_{i=1}^{n} y_{i} e_{i}$, where $Y=\left(y_{1}, \ldots, y_{n}\right)^{T} \in K^{n}$, we have

$$
\partial(m)=\sum_{i=1}^{n} \partial\left(y_{i}\right) e_{i}-\sum_{i=1}^{n}\left(\sum_{j=1}^{n} a_{i, j} y_{j}\right) e_{i} .
$$

Thus, the equation $\partial(m)=0$ translates into the linear differential system $\partial(Y)=A Y$.

Definition 2.33 Let $\mathscr{M}$ be a $\partial$-module over $K$ and $\left\{e_{1}, \ldots, e_{n}\right\}$ be a $K$-basis of $\mathscr{M}$. We say that the linear differential system $\partial(Y)=A Y$, as above, is associated to the $\partial$-module $\mathscr{M}$ (via the choice of a $K$-basis). Conversely, to a given linear differential system $\partial(Y)=A Y, A=\left(a_{i, j}\right) \in K^{n \times n}$, one associates a $\partial$-module $\mathscr{M}$ over $K$, namely $\mathscr{M}=K^{n}$ with the standard basis $\left(e_{1}, \ldots, e_{n}\right)$ and action of $\partial$ given by (2.1).

Another choice of a $K$-basis $X=B Y$, where $B \in \mathrm{GL}_{n}(K)$, leads to the differential system

$$
\partial(X)=\left(B^{-1} A B-B^{-1} \partial(B)\right) X
$$

Definition 2.34 We say that a linear differential system $\partial(X)=\tilde{A} X$, with $\tilde{A} \in K^{n \times n}$, is $K$-equivalent (or gauge equivalent over $K$ ) to a linear differential system $\partial(X)=A X$, with $A \in K^{n \times n}$, if there exists $B \in \mathrm{GL}_{n}(K)$ such that

$$
\tilde{A}=B^{-1} A B-B^{-1} \partial(B) .
$$

One has the following correspondence between linear differential systems and linear differential equations. For $L=\partial^{n}+a_{n-1} \partial^{n-1}+\ldots+a_{0} \in K[\partial]$, one can consider the companion matrix

$$
A_{L}=\left(\begin{array}{cccc}
0 & 1 & \ldots & 0 \\
0 & \ddots & \ddots & \vdots \\
\vdots & \ddots & 0 & 1 \\
-a_{0} & -a_{1} & \ldots & -a_{n-1}
\end{array}\right) \text {. }
$$


The differential system $\partial Y=A_{L} Y$ induces a $\partial$-module structure on $K^{n}$, which we denote by $\mathscr{L}$. Conversely, the Cyclic vector lemma [45, Proposition 2.9] states that any $\partial$-module is isomorphic to a $\partial$-module $\mathscr{L}$, of the above form, provided $\mathbf{k} \subsetneq K$.

Definition 2.35 A morphism of $\partial$-modules over $K$ is a homomorphism of $K[\partial]$ modules.

One can consider the category $\operatorname{Diff}_{K}$ of $\partial$-modules over $K$ :

Definition 2.36 We can define the following constructions in $\operatorname{Diff}_{K}$ :

(1) The direct sum of two $\partial$-modules, $\mathscr{M}_{1}$ and $\mathscr{M}_{2}$, is $\mathscr{M}_{1} \oplus \mathscr{M}_{2}$ together with the action of $\partial$ defined by

$$
\partial\left(m_{1} \oplus m_{2}\right)=\partial\left(m_{1}\right) \oplus \partial\left(m_{2}\right) .
$$

(2) The tensor product of two $\partial$-modules, $\mathscr{M}_{1}$ and $\mathscr{M}_{2}$, is $\mathscr{M}_{1} \otimes_{K} \mathscr{M}_{2}$ together with the action of $\partial$ defined by

$$
\partial\left(m_{1} \otimes m_{2}\right)=\partial\left(m_{1}\right) \otimes m_{2}+m_{1} \otimes \partial\left(m_{2}\right) .
$$

(3) The unit object $\mathbf{1}$ for the tensor product is the field $K$ together with the left $K[\partial]$ module structure given by

$$
\left(a_{0}+a_{1} \partial+\cdots+a_{n} \partial^{n}\right)(f)=a_{0} f+\cdots+a_{n} \partial^{n}(f)
$$

for $f, a_{0}, \ldots, a_{n} \in K$.

(4) The internal Hom of two $\partial$-modules $\mathscr{M}_{1}, \mathscr{M}_{2}$ exists in $\operatorname{Diff}_{K}$ and is denoted by $\underline{\operatorname{Hom}}\left(\mathscr{M}_{1}, \mathscr{M}_{2}\right)$. It consists of the $K$-vector space $\operatorname{Hom}_{K}\left(\mathscr{M}_{1}, \mathscr{M}_{2}\right)$ of $K$-linear maps from $\mathscr{M}_{1}$ to $\mathscr{M}_{2}$ together with the action of $\partial$ given by the formula

$$
\partial u\left(m_{1}\right)=\partial\left(u\left(m_{1}\right)\right)-u\left(\partial m_{1}\right) .
$$

The dual $\mathscr{M}^{*}$ of a $\partial$-module $\mathscr{M}$ is the $\partial$-module $\underline{\operatorname{Hom}}(\mathscr{M}, \mathbf{1})$.

(5) An endofunctor $D:$ Diff $_{K} \rightarrow$ Diff $_{K}$, called the prolongation functor, is defined as follows: if $\mathscr{M}$ is an object of $\operatorname{Diff}_{K}$ corresponding to the linear differential system $\partial(Y)=A Y$, then $D(\mathscr{M})$ corresponds to the linear differential system

$$
\partial(Z)=\left(\begin{array}{cc}
A & \delta(A) \\
0 & A
\end{array}\right) Z .
$$

The construction of the prolongation functor reflects the following idea. If $U$ is a fundamental solution matrix of $\partial(Y)=A Y$ in some $\Delta$-field extension $F$ of $K$, that is, $\partial(U)=A U$ and $U \in \mathrm{GL}_{n}(F)$, then

$$
\partial(\delta U)=\delta(\partial U)=\delta(A) U+A \delta(U) .
$$

Then, $\left(\begin{array}{cc}U & \delta(U) \\ 0 & U\end{array}\right)$ is a fundamental solution matrix of $\partial(Z)=\left(\begin{array}{cc}A & \delta(A) \\ 0 & A\end{array}\right) Z$. Endowed with all these constructions, it follows from [44, Corollary 3] that the category $\operatorname{Diff}_{K}$ is a $\delta$-tensor category (in the sense of [44, Definition 3] and [26, Definition 4.2.1]).

In this paper, we will not consider the whole category Diff ${ }_{K}$ but the $\delta$-tensor subcategory generated by a $\partial$-module. More precisely, we have the following definition. 
Definition 2.37 Let $\mathscr{M}$ be an object of $\operatorname{Diff}_{K}$. We denote by $\{\mathscr{M}\}^{\otimes, \delta}$ the smallest full subcategory of $\operatorname{Diff}_{K}$ that contains $\mathscr{M}$ and is closed under all operations of linear algebra (direct sums, tensor products, duals, and subquotients) and under $D$. The category $\{\mathscr{M}\}^{\otimes, \delta}$ is a $\delta$-tensor category over $\mathbf{k}$. We also denote by $\{\mathscr{M}\}^{\otimes}$ the full tensor subcategory of $\operatorname{Diff}_{K}$ generated by $\mathscr{M}$. Then, $\{\mathscr{M}\}^{\otimes}$ is a tensor category over k.

Similarly, the category Vect $\mathbf{k}_{\mathbf{k}}$ of finite-dimensional $\mathbf{k}$-vector spaces is a $\delta$-tensor category. The prolongation functor on $\mathbf{V e c t}_{\mathbf{k}}$ is defined as follows: for a $\mathbf{k}$-vector space $V$, the k-vector space $D(V)$ equals $\mathbf{k}[\delta]_{\leq 1} \otimes_{\mathbf{k}} V$, where $\mathbf{k}[\delta]_{\leq 1}$ is considered as the right $\mathbf{k}$-module of $\delta$-operators up to order 1 and $V$ is viewed as a left $\mathbf{k}$-module.

Definition 2.38 Let $\mathscr{M}$ be an object of Diff ${ }_{K}$. A $\delta$-fiber functor $\omega:\{\mathscr{M}\}^{\otimes, \delta} \rightarrow$ Vect $_{\mathbf{k}}$ is an exact, faithful, k-linear, tensor compatible functor together with a natural isomorphism between $D_{\text {Vect }_{\mathbf{k}}} \circ \omega$ and $\omega \circ D_{\{\mathscr{M}\}} \otimes, \delta$ [26, Definition 4.2.7], where the subscripts emphasize the category on which we perform the prolongation. The pair $\left(\{\mathscr{M}\}^{\otimes, \delta}, \omega\right)$ is called a $\delta$-Tannakian category.

Theorem 2.39 ([20, Corollaries 4.29 and 6.2]) Let $\mathscr{M}$ be an object of Diff $_{K}$. Since $\mathbf{k}$ is $\delta$-closed, the category $\{\mathscr{M}\}^{\otimes, \delta}$ admits a $\delta$-fiber functor and any two $\delta$-fiber functors are naturally isomorphic.

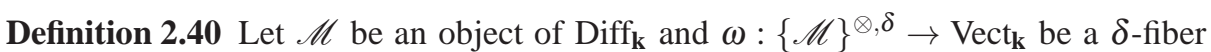
functor. The group $\operatorname{Gal}^{\delta}(\mathscr{M})$ of $\delta$-tensor isomorphisms of $\omega$ is defined as follows. It consists of the elements $g \in \operatorname{GL}(\omega(\mathscr{M}))$ that stabilize $\omega(\mathscr{V})$ for every $\partial$-module $\mathscr{V}$ obtained from $\mathscr{M}$ by applying the linear constructions (subquotient, direct sum, tensor product, and dual), and the prolongation functor. The action of $g$ on $\omega(\mathscr{V})$ is obtained by applying the same constructions to $g$. We call $\mathrm{Gal}^{\delta}(\mathscr{M})$ the parameterized differential Galois group of $(\mathscr{M}, \omega)$, or of $\mathscr{M}$ when there is no confusion.

Theorem 2.41 ([44, Theorem 2]) Let $\mathscr{M}$ be an object of $\operatorname{Diff}_{K}$ and $\omega:\{\mathscr{M}\}^{\otimes, \delta} \rightarrow$ Vect $\mathrm{k}_{\mathbf{k}}$ be a $\delta$-fiber functor. The group $\mathrm{Gal}^{\delta}(\mathscr{M}) \subset \mathrm{GL}(\omega(\mathscr{M}))$ is a linear differential algebraic group defined over $\mathbf{k}$, and $\omega$ induces an equivalence of categories between $\{\mathscr{M}\}^{\otimes, \delta}$ and the category of finite-dimensional representations of $\mathrm{Gal}^{\delta}(\mathscr{M})$.

Definition 2.42 We say that a $\partial$-module $\mathscr{M}$ over $K$ is trivial if it is either $(0)$ or isomorphic as $\partial$-module over $K$ to $\mathbf{1}^{n}$ for some positive integer $n$. For $G$ a linear differential algebraic group over $\mathbf{k}$, we say that a $G$-module $V$ is trivial if $G$ acts identically on $V$.

Remark 2.43 For $\mathscr{M}$ an object of $\operatorname{Diff}_{K}$ and $\omega:\{\mathscr{M}\}^{\otimes, \delta} \rightarrow$ Vect $_{\mathbf{k}}$ a $\delta$-fiber functor, the following holds: a $\partial$-module $\mathscr{N}$ in $\{\mathscr{M}\}^{\otimes, \delta}$ is trivial if and only if $\omega(\mathscr{N})$ is a trivial $\mathrm{Gal}^{\delta}(\mathscr{M})$-module.

Remark 2.44 The parameterized differential Galois group depends a priori on the choice of a $\delta$-fiber functor $\omega$. However, since two $\delta$-fiber functors for $\{\mathscr{M}\}^{\otimes, \delta}$ are naturally isomorphic, we find that the parameterized differential Galois groups that these functors define are isomorphic as linear differential algebraic groups over k. Thus, if it is not necessary, we will speak of the parameterized differential Galois group of $\mathscr{M}$ without mentioning the $\delta$-fiber functor. 
Forgetting the action of $\delta$, one can similarly define the group $\operatorname{Gal}(\mathscr{M})$ of tensor isomorphisms of $\omega:\{\mathscr{M}\}^{\otimes} \rightarrow \operatorname{Vect}_{\mathbf{k}}$. By [14], the group $\operatorname{Gal}(\mathscr{M}) \subset \operatorname{GL}(\omega(\mathscr{M}))$ is a linear algebraic group defined over $\mathbf{k}$, and $\omega$ induces an equivalence of categories between $\{\mathscr{M}\}^{\otimes}$ and the category of k-finite-dimensional representations of $\operatorname{Gal}(\mathscr{M})$. We call $\mathrm{Gal}(\mathscr{M})$ the differential Galois group of $\mathscr{M}$ over $K$.

Proposition 2.45 ([22, Proposition 6.21]) If $\mathscr{M}$ is an object of $\operatorname{Diff}_{K}$ and $\omega$ : $\{\mathscr{M}\}^{\otimes, \delta} \rightarrow \operatorname{Vect}_{\mathbf{k}}$ is a $\delta$-fiber functor, then $\mathrm{Gal}^{\delta}(\mathscr{M})$ is a Zariski dense subgroup of $\mathrm{Gal}(\mathscr{M})$ (see Proposition 2.13).

Definition 2.46 A parameterized Picard-Vessiot extension, or PPV extension for short, of $K$ for a $\partial$-module $\mathscr{M}$ over $K$ is a $\Delta$-field extension $K_{\mathscr{M}}$ that is generated over $K$ by the entries of a fundamental solution matrix $U$ of a differential system $\partial(X)=A X$ associated to $\mathscr{M}$ and such that $K_{\mathscr{M}}^{\partial}=K^{\partial}$. The field $K(U)$ is a Picard-Vessiot extension ( $P V$ extension for short), that is, a $\partial$-field extension of $K$ generated by the entries of a fundamental solution matrix $U$ of $\partial(X)=A X$ such that $K(U)^{\partial}=K^{\partial}$.

A parameterized Picard-Vessiot extension associated to a $\partial$-module $\mathscr{M}$ depends a priori on the choice of a $K$-basis of $\mathscr{M}$, which is equivalent to the choice of a linear differential system associated to $\mathscr{M}$. However, one can show that gauge equivalent differential systems lead to parameterized Picard-Vessiot extensions that are isomorphic as $K$ - $\Delta$-algebras. In [14], Deligne showed that a fiber functor corresponds to a PicardVessiot extension; it is shown in [20, Theorem 5.5] that the notions of $\delta$-fiber functor and parameterized Picard-Vessiot extension are equivalent.

Definition 2.47 Let $\mathscr{M}$ be a $\partial$-module over $K$. Let $\partial(X)=A X$ be a differential system associated to $\mathscr{M}$ over $K$ with $A \in K^{n \times n}$ and let $K_{\mathscr{M}}$ be a PPV extension for $\partial(X)=A X$ over $K$. The parameterized Picard-Vessiot group, or PPV-group for short is denoted by $\mathrm{Gal}^{\delta}\left(K_{\mathscr{M}} / K\right)$ and is the set of $\Delta$-automorphisms of $K_{\mathscr{M}}$ over $K$, whereas the PicardVessiot group (usually called the differential Galois group in the literature) of $K_{\mathscr{M}}$ over $K$, by definition, is the set of $\partial$-automorphisms of a Picard-Vessiot extension $K(U)$ of $K$ in $K_{\mathscr{M}}$, where $U \in \mathrm{GL}_{n}\left(K_{\mathscr{M}}\right)$ is a fundamental solution matrix of $\partial(X)=A X$. This group is denoted by $\operatorname{Gal}\left(K_{\mathscr{M}} / K\right)$.

Remark 2.48 Let $U \in \mathrm{GL}_{n}\left(K_{\mathscr{M}}\right)$ be a fundamental solution matrix of $\partial(X)=A X$. For any $\tau \in \operatorname{Gal}^{\delta}\left(K_{\mathscr{M}} / K\right)$, there exists $[\tau]_{U} \in \mathrm{GL}_{n}(\mathbf{k})$ such that $\tau(U)=U[\tau]_{U}$. The map

$$
\operatorname{Gal}^{\delta}\left(K_{\mathscr{M}} / K\right) \rightarrow \mathrm{GL}_{n}, \quad \tau \mapsto[\tau]_{U}
$$

is an embedding and identifies $\mathrm{Gal}^{\delta}\left(K_{\mathscr{M}} / K\right)$ with a $\delta$-closed subgroup of $\mathrm{GL}_{n}$. One can show that another choice of fundamental solution matrix as well as another choice of gauge equivalent linear differential system yield a conjugate subgroup in $\mathrm{GL}_{n}$. Similarly, one can represent $\operatorname{Gal}\left(K_{\mathscr{M}} / K\right)$ as a linear algebraic subgroup of $\mathrm{GL}_{n}$. With these representations of the Picard-Vessiot groups, one can show that Picard-Vessiot groups and differential Galois groups are isomorphic in the parameterized and nonparameterized cases. 
In the PPV theory, a Galois correspondence holds between differential algebraic subgroups of the PPV-group and $\Delta$-sub-field extensions of $K_{\mathscr{M}}$ (see [22, Theorem 6.20] for more details). Moreover, the $\delta$-dimension of $\mathrm{Gal}^{\delta}(\mathscr{M})$ coincides with the $\delta$ transcendence degree of $K_{\mathscr{M}}$ over $K$ (see [22, page 374 and Proposition 6.26] for the definition of the $\delta$-dimension and $\delta$-transcendence degree and the proof of their equality). Moreover, the defining equations of the parameterized differential Galois group reflect the differential algebraic relations among the solutions (see [22, Proposition 6.24]). Therefore, given a $\partial$-module $\mathscr{M}$ over $K$, we find that the defining equations of the parameterized differential Galois group $\operatorname{Gal}^{\delta}(\mathscr{M})$ over k determine the differential algebraic relations between the solutions in $K_{\mathscr{M}}$ over $K$.

Definition 2.49 A $\partial$-module $\mathscr{M}$ is said to be completely reducible if, for every $\partial$ submodule $\mathscr{N}$ of $\mathscr{M}$, there exists a $\partial$-submodule $\mathscr{N}^{\prime}$ of $\mathscr{M}$ such that $\mathscr{M}=\mathscr{N} \oplus$ $\mathscr{N}^{\prime}$. We say that a $\partial$-operator is completely reducible if the associated $\partial$-module is completely reducible.

By [45, Exercise 2.38], a $\partial$-module is completely reducible if and only if its differential Galois group is a reductive linear algebraic group. Moreover, for a completely reducible $\partial$-module $\mathscr{M}$, any object in $\{\mathscr{M}\}^{\otimes}$ is completely reducible.

\subsubsection{Isomonodromic differential modules}

Definition 2.50 ([13, Definition 3.8]) Let $A \in K^{n \times n}$. We say that the linear differential system $\partial Y=A Y$ is isomonodromic (or completely integrable) over $K$ if there exists $B \in K^{n \times n}$ such that

$$
\partial(B)-\delta(A)=A B-B A .
$$

Remark 2.51 One can show that a linear differential system $\partial Y=A Y$ is isomonodromic if and only if there exists a $\Delta$-field extension $L$ of $K$ and $B \in K^{n \times n}$ such that the system

$$
\left\{\begin{array}{l}
\partial Y=A Y \\
\delta Y=B Y
\end{array}\right.
$$

has a fundamental solution matrix with coefficients in $L$.

We recall a characterization of complete integrability in terms of the PPV theory.

Proposition 2.52 ([13, Proposition 3.9]) Let $\mathscr{M}$ be a $\partial$-module over $K$ and $\partial(Y)=$ $A Y$, with $A \in K^{n \times n}$, be an associated linear differential system. The following statements are equivalent:

- $\mathrm{Gal}^{\delta}(\mathscr{M})$ is conjugate to constants in $\mathrm{GL}(\omega(\mathscr{M}))$ (see Definition 2.30);

- The linear differential system $\partial(Y)=A Y$ is isomonodromic over $K$.

The proof of the following result was provided to the authors by Michael F. Singer and will be used in the proof of Proposition 2.54. 
Lemma 2.53 Given a linear differential algebraic group $G \subset \mathrm{GL}_{n}$ defined over a differentially closed field $(\mathbf{k}, \delta)$ and any $\Delta=\{\partial, \delta\}$-field $K$ such that $K^{\partial}=\mathbf{k}$, there exists $a \Delta$-field extension $F$ of $K$ such that $F^{\partial}=\mathbf{k}$ and $G$ can be realized as a parameterized differential Galois group over $F$ in the given faithful representation of $G \subset \mathrm{GL}_{n}$.

Proof We first consider the "generic" case: we construct a $\Delta$-field extension $E$ of $K$ with no new $\partial$-constants such that $\mathrm{GL}_{n}$ is a parameterized differential Galois group of a $\partial$-module $\mathscr{M}$ over $E$. Assume we have constructed $E$ and let $E_{\mathscr{M}}$ be a PPV extension of $\mathscr{M}$ over $E$. For any differential algebraic subgroup $G$ of $\mathrm{GL}_{n}$, let $F$ be the fixed field of $G$ in $E_{\mathscr{M}}$, i.e., the elements of $E_{\mathscr{M}}$ fixed by $G$. By the PPV correspondence, $G$ is the parameterized differential Galois group of $E_{\mathscr{M}}$ over $F$. Moreover,

$$
K^{\partial}=\mathbf{k} \subset F^{\partial} \subset E_{\mathscr{M}}^{\partial}=\mathbf{k} .
$$

To construct the fields $E_{\mathscr{M}}$ and $E$ for $\mathrm{GL}_{n}$, we shall follow the construction from [31, pages 87-89]. Let $\left\{z_{i, j}\right\}$ be a set of $n^{2} \Delta$-differential indeterminates over $K$. Let $E_{\mathscr{M}}=$ $K\left\langle z_{i, j}\right\rangle_{\Delta}$ be a $\Delta$-field of differential rational functions in these indeterminates. Note that the $\delta$-constants of $E_{\mathscr{M}}$ are $\mathbf{k}$, as in [31, Lemma 2.14]. Let $Z=\left(z_{i, j}\right) \in \mathrm{GL}_{n}\left(E_{\mathscr{M}}\right)$ and $A=(\partial Z)(Z)^{-1}$. We then have that

$$
\partial Z=A Z .
$$

Let $E$ be the $\Delta$-field generated over $K$ by the entries of $A$. Then, $E_{\mathscr{M}}$ is a PPV extension of $E$ for equation (2.2). Since $Z$ is a matrix of $\Delta$-differential indeterminates, any assignment $Z \mapsto Z g$ for $g \in \mathrm{GL}_{n}(K)$ defines a $\Delta$ - $K$-automorphism $\phi_{g}$ of $E_{\mathscr{M}}$ over $K$. If we restrict to those $g \in \mathrm{GL}_{n}=\mathrm{GL}_{n}(\mathbf{k})$, then $\phi_{g}$ leaves $A$ fixed and so all elements of $E$ are left fixed. Therefore, $\mathrm{GL}_{n}$ is a subgroup of the PPV-group of $E_{\mathscr{M}}$ over $E$. Since this PPV-group is already a subgroup of $\mathrm{GL}_{n}$, we must have that the PPV-group of $E_{\mathscr{M}}$ over $E$ is $\mathrm{GL}_{n}$.

The proof of the following result uses PPV theory, which does not appear in the statement. It is, therefore, of interest to find a direct proof of it as well.

Proposition 2.54 Let $G \subset \mathrm{GL}(V)$ be a linear differential algebraic group over $\mathbf{k}$ and let $G^{\circ}$ be the identity component of $G$. If $G^{\circ}$ is conjugate to constants in $\operatorname{GL}(V)$, then the same holds for $G$.

Proof By Lemma 2.53, let $K$ be a $\Delta$-field with $K^{\partial}=\mathbf{k}$ such that $G$ is a parameterized differential Galois group of a $\partial$-module $\mathscr{M}$ over $K$ and the embedding $G \subset \mathrm{GL}(V)$ is the faithful representation $G \rightarrow \operatorname{GL}(\omega(\mathscr{M}))$. Let $L / K$ be a PPV extension for $\mathscr{M}$ over $K$. One can identify $G$ with $\mathrm{Gal}^{\delta}(L / K)$, the group of automorphisms of $L$ over $K$ commuting with $\delta$ and $\partial$. Let $F$ be the subfield of $L$ fixed by $G^{\circ}$. By the PPV correspondence [13, Theorem 9.5], the group of automorphisms of $L$ over $F$ commuting with $\{\delta, \partial\}$ coincides with $G^{\circ}$ and the extension $F / K$ is algebraic since $G / G^{\circ}$ is finite.

Let $\partial(Y)=A Y$ be a linear differential system associated to $\mathscr{M}$. The parameterized differential Galois group of $\mathscr{M}$ over $F$ is $G^{\circ}$ and thus conjugate to constants by assumption. Proposition 2.52 implies that $\partial(Y)=A Y$ is isomonodromic over $F$, that is, there exists $B \in F^{n \times n}$ such that

$$
\partial(B)-\delta(A)=A B-B A .
$$


Let $K_{0}$ be the subfield extension of $F$ generated over $K$ by the coefficients of the matrix $B$. Without loss of generality, we can assume that $K_{0} / K$ is a finite Galois extension in the classical sense. We denote by $\operatorname{Gal}\left(K_{0} / K\right)$ its differential Galois group and by $r$ its degree. By [45, Exercise 1.24], there exist unique derivations, still denoted $\partial$ and $\delta$ extending $\partial$ and $\delta$ to $K_{0}$. Moreover, any element of $\operatorname{Gal}\left(K_{0} / K\right)$ commutes with the action of $\delta$ and $\partial$ on $K_{0}$. If we let

$$
C=\frac{1}{r} \sum_{\tau \in \mathrm{Gal}\left(K_{0} / K\right)} \tau(B),
$$

then $C$ has coefficients in $K$ and satisfies

$$
\begin{aligned}
& \partial(A)-\delta(C)=\partial(A)-\frac{1}{r}\left(\sum_{\tau \in \operatorname{Gal}\left(K_{0} / K\right)} \tau(\delta(B))\right) \\
& =\partial(A)-\frac{1}{r}\left(\sum_{\tau \in \operatorname{Gal}\left(K_{0} / K\right)} \tau(\partial(A)-B A+A B)\right)=\partial(A)-\partial(A)+C A-A C .
\end{aligned}
$$

This shows that $\partial(Y)=A Y$ is isomonodromic over $K$. By Proposition 2.52, we find that $G$ is conjugate to constants in $\mathrm{GL}_{n}$.

\section{Calculating the parameterized differential Galois group of $L_{1}\left(L_{2}(y)\right)=0$}

In this section, given two completely reducible $\partial$-modules $\mathscr{L}_{1}$ and $\mathscr{L}_{2}$, we study the parameterized differential Galois group of an arbitrary $\partial$-module extension $\mathscr{U}$ of $\mathscr{L}_{1}$ by $\mathscr{L}_{2}$. In Section 3.1, we describe $\mathrm{Gal}^{\delta}(\mathscr{U})$ as a semi-direct product of a $\delta$-closed subgroup of $\operatorname{Hom}\left(\omega\left(\mathscr{L}_{1}\right), \omega\left(\mathscr{L}_{2}\right)\right)$ by the parameterized differential Galois group $\mathrm{Gal}^{\delta}\left(\mathscr{L}_{1} \oplus \mathscr{L}_{2}\right)$ (see Theorem 3.3). In Section 3.2, we perform a first reduction that allows us to set $\mathscr{L}_{1}$ equal to the trivial $\partial$-module 1 .

In Theorem 3.13, we show how one can recover a complete description of the parametrized differential Galois group of $\mathscr{U}$ from the knowledge of the parametrized differential Galois group of its reduction. In Section 3.3, we thus focus on the computation of the parameterized differential Galois group of an arbitrary $\partial$-module extension $\mathscr{U}$ of 1 by a completely reducible $\partial$-module $\mathscr{L}$.

We then show that one can decompose $\mathscr{L}$ in a "constant" and a "purely nonconstant" part. This decomposition yields a decomposition of $R_{u}\left(\mathrm{Gal}^{\delta}(\mathscr{U})\right)$. For $K=$ $\mathbf{k}(x)$, the computation of $\mathrm{Gal}^{\delta}(\mathscr{U})$ for the "constant part" can be deduced from the algorithms contained in [35], whereas the computation of the "purely non-constant" part results from Section 3.3.2 and Theorem 3.19. Finally, in Section 3.3.3, we show, under some assumption on $\mathscr{L}$, that $R_{u}\left(\mathrm{Gal}^{\delta}(\mathscr{U})\right)$ is the product of the "constant" and "purely non-constant" parts (see Theorem 3.25).

Throughout this section, $K$ is a $(\delta, \partial)$-field of characteristic zero, whose field of $\partial$-constants $\mathbf{k}$ is assumed to be $\delta$-closed. We denote also by $C$ the field of $\delta$-constants of k. We fix a $\delta$-fiber functor $\omega: \operatorname{Diff}_{K} \rightarrow \operatorname{Vect}_{\mathbf{k}}$ on $\operatorname{Diff}_{K}$ (see Definition 2.38). Any parameterized differential Galois group in this section shall be computed with respect 
to $\omega$ and is a linear differential algebraic group defined over k. Any representation is, unless explicitly mentioned, defined over $\mathbf{k}$.

\subsection{Structure of the parameterized differential Galois group}

Let $L_{1}, L_{2} \in K[\partial]$ be two completely reducible $\partial$-operators, and let us denote by $\mathscr{L}_{1}$ (respectively, by $\mathscr{L}_{2}$ ) the $\partial$-module corresponding to $L_{1}(y)=0$ (respectively, $L_{2}(y)=$ 0 ). The $\partial$-module $\mathscr{U}$ over $K$, corresponding to $L_{1}\left(L_{2}(y)\right)=0$, is an extension of $\mathscr{L}_{1}$ by $\mathscr{L}_{2}$,

$$
0 \longrightarrow \mathscr{L}_{2} \stackrel{i}{\longrightarrow} \mathscr{U} \stackrel{p}{\longrightarrow} \mathscr{L}_{1} \longrightarrow 0
$$

in the category of $\partial$-modules over $K$.

Definition 3.1 For any object $\mathscr{X}$ in $\{\mathscr{U}\}^{\otimes, \delta}$, we define $\operatorname{Stab}(\mathscr{X})$ (respectively, $\operatorname{Stab}^{\delta}(\mathscr{X})$ ) as the set of (respectively, $\delta$-) tensor automorphisms in $\operatorname{Gal}(\mathscr{U})$ (respectively, $\left.\mathrm{Gal}^{\delta}(\mathscr{U})\right)$ that induce the identity on $\omega(\mathscr{X})$.

By [15, II.1.36], $\operatorname{Stab}(\mathscr{X})$ (respectively, $\operatorname{Stab}^{\delta}(\mathscr{X})$ ) is a linear (respectively, differential) algebraic group over k. One has also that $\operatorname{Stab}^{\delta}(\mathscr{X})$ is Zariski dense in $\operatorname{Stab}(\mathscr{X})$. Moreover, we have:

Lemma 3.2 For any object $\mathscr{X}$ in $\{\mathscr{U}\}^{\otimes, \delta}$, the group $\operatorname{Stab}^{\delta}(\mathscr{X})$ (respectively, $\operatorname{Stab}(\mathscr{X})$ ) is normal in $\mathrm{Gal}^{\delta}(\mathscr{U})$ (respectively, $\operatorname{Gal}(\mathscr{U})$ ).

Proof We prove only the parameterized statement. Let $g \in \mathrm{Gal}^{\delta}(\mathscr{U})$ and $h \in$ $\operatorname{Stab}^{\delta}(\mathscr{X})$. One has to show that $g h g^{-1}$ induces the identity on $\omega(\mathscr{X})$. It is sufficient to remark that, by definition, any element of $\mathrm{Gal}^{\delta}(\mathscr{U})$ stabilizes $\omega(\mathscr{X})$.

The aim of this section is to prove the following theorem.

Theorem 3.3 If $\mathscr{L}_{1}, \mathscr{L}_{2}$ are completely reducible $\partial$-modules over $K$ and if $\mathscr{U}$ is a $\partial$ module extension over $K$ of $\mathscr{L}_{1}$ by $\mathscr{L}_{2}$, then

(1) $\operatorname{Gal}^{\delta}(\mathscr{U})$ is an extension of $\operatorname{Gal}^{\delta}\left(\mathscr{L}_{1} \oplus \mathscr{L}_{2}\right)$ by a $\delta$-subgroup $W \subset$ $\operatorname{Hom}\left(\omega\left(\mathscr{L}_{1}\right), \omega\left(\mathscr{L}_{2}\right)\right)$

(2) $W$ is stable under the action of $\mathrm{Gal}^{\delta}\left(\mathscr{L}_{1} \oplus \mathscr{L}_{2}\right)$ on $\operatorname{Hom}\left(\omega\left(\mathscr{L}_{1}\right), \omega\left(\mathscr{L}_{2}\right)\right)$ given by

$$
g * \phi=g \phi\left(g^{-1}\right) \quad \text { for any }(g, \phi) \in \mathrm{Gal}^{\delta}\left(\mathscr{L}_{1} \oplus \mathscr{L}_{2}\right) \times \operatorname{Hom}\left(\omega\left(\mathscr{L}_{1}\right), \omega\left(\mathscr{L}_{2}\right)\right) .
$$

Remark 3.4 The parameterized differential Galois group $\mathrm{Gal}^{\delta}\left(\mathscr{L}_{1} \oplus \mathscr{L}_{2}\right)$ acts on the objects of the $\delta$-tensor category generated by $\omega\left(\mathscr{L}_{1} \oplus \mathscr{L}_{2}\right)$. The k-vector space $\operatorname{Hom}\left(\omega\left(\mathscr{L}_{1}\right), \omega\left(\mathscr{L}_{2}\right)\right)$ belongs to this category, and the action of $\mathrm{Gal}^{\delta}\left(\mathscr{L}_{1} \oplus \mathscr{L}_{2}\right)$ on $\operatorname{Hom}\left(\omega\left(\mathscr{L}_{1}\right), \omega\left(\mathscr{L}_{2}\right)\right)$ detailed above is just the description of the Tannakian representation.

Before proving this theorem, we need some intermediate lemmas. 
Lemma 3.5 The linear differential algebraic group $\mathrm{Gal}^{\delta}(\mathscr{U})$ is an extension of the reductive linear differential algebraic group $\mathrm{Gal}^{\delta}\left(\mathscr{L}_{1} \oplus \mathscr{L}_{2}\right)$ by the linear differential algebraic group $\operatorname{Stab}^{\delta}\left(\mathscr{L}_{1} \oplus \mathscr{L}_{2}\right)$.

Proof Since $\left\{\mathscr{L}_{1} \oplus \mathscr{L}_{2}\right\}^{\otimes, \delta}$ is a full $\delta$-tensor subcategory of $\{\mathscr{U}\}^{\otimes, \delta}$, the linear differential algebraic group $\mathrm{Gal}^{\delta}\left(\mathscr{L}_{1} \oplus \mathscr{L}_{2}\right)$ is a quotient of $\mathrm{Gal}^{\delta}(\mathscr{U})$. We denote the quotient map by

$$
\pi: \mathrm{Gal}^{\delta}(\mathscr{U}) \rightarrow \mathrm{Gal}^{\delta}\left(\mathscr{L}_{1} \oplus \mathscr{L}_{2}\right)
$$

Then ker $\pi=\operatorname{Stab}^{\delta}\left(\mathscr{L}_{1} \oplus \mathscr{L}_{2}\right)$. Since $\mathscr{L}_{1}$ and $\mathscr{L}_{2}$ are completely reducible, $\mathscr{L}_{1} \oplus \mathscr{L}_{2}$ is completely reducible as well. This means that $\mathrm{Gal}^{\delta}\left(\mathscr{L}_{1} \oplus \mathscr{L}_{2}\right)$ is reductive. Since the latter group is the Zariski closure of $\mathrm{Gal}^{\delta}\left(\mathscr{L}_{1} \oplus \mathscr{L}_{2}\right)$ in $\mathrm{GL}\left(\omega\left(\mathscr{L}_{1} \oplus \mathscr{L}_{2}\right)\right)$, [36, Remark 2.9] implies that $\mathrm{Gal}^{\delta}\left(\mathscr{L}_{1} \oplus \mathscr{L}_{2}\right)$ is a reductive linear differential algebraic group.

We will relate $\operatorname{Stab}^{\delta}\left(\mathscr{L}_{1} \oplus \mathscr{L}_{2}\right)$ to $R_{u}\left(\mathrm{Gal}^{\delta}(\mathscr{U})\right)$ and describe more precisely the structure of the latter group. By the exactness of $\omega, \omega(\mathscr{U})$ is an extension of $\omega\left(\mathscr{L}_{1}\right)$ by $\omega\left(\mathscr{L}_{2}\right)$ in the category of representations of $\mathrm{Gal}^{\delta}(\mathscr{U})$.

Lemma 3.6 In the above notation, let s be a $\mathbf{k}$-linear section of the exact sequence:

$$
0 \longrightarrow \omega\left(\mathscr{L}_{2}\right) \stackrel{\omega(i)}{\longrightarrow} \omega(\mathscr{U}) \underset{\gtrless_{s} \ldots}{\stackrel{\omega(p)}{\gtrless}} \omega\left(\mathscr{L}_{1}\right) \longrightarrow 0
$$

We consider the following map

$$
\zeta_{\mathscr{U}}: \operatorname{Gal}^{\delta}(\mathscr{U}) \rightarrow \operatorname{Hom}\left(\omega\left(\mathscr{L}_{1}\right), \omega\left(\mathscr{L}_{2}\right)\right), \quad g \mapsto\left(x \mapsto g\left(s\left(g^{-1} x\right)\right)-s(x)\right) .
$$

Then the restriction of the map $\zeta_{\mathscr{U}}$ to $\operatorname{Stab}^{\delta}\left(\mathscr{L}_{1} \oplus \mathscr{L}_{2}\right)$ is a one-to-one morphism of linear differential algebraic groups. Moreover, the linear differential algebraic group $\operatorname{Stab}^{\delta}\left(\mathscr{L}_{1} \oplus \mathscr{L}_{2}\right)$ is abelian and coincides with $R_{u}\left(\mathrm{Gal}^{\delta}(\mathscr{U})\right)$.

Proof For all $g_{1}, g_{2} \in \mathrm{Gal}^{\delta}(\mathscr{U})$, we have:

$$
\zeta_{\mathscr{U}}\left(g_{1} g_{2}\right)(x)=g_{1} \zeta_{\mathscr{U}}\left(g_{2}\right)\left(g_{1}^{-1} x\right)+\zeta_{\mathscr{U}}\left(g_{1}\right)(x) .
$$

If $g_{1}, g_{2} \in \operatorname{Stab}^{\delta}\left(\mathscr{L}_{1} \oplus \mathscr{L}_{2}\right)$, equation (3.2) gives

$$
\zeta_{\mathscr{U}}\left(g_{1} g_{2}\right)=\zeta_{\mathscr{U}}\left(g_{1}\right)+\zeta_{\mathscr{U}}\left(g_{2}\right) .
$$

This means that $\zeta_{\mathscr{U}}$ is a morphism of linear differential algebraic groups from $\operatorname{Stab}^{\delta}\left(\mathscr{L}_{1} \oplus \mathscr{L}_{2}\right)$ to $\operatorname{Hom}\left(\omega\left(\mathscr{L}_{1}\right), \omega\left(\mathscr{L}_{2}\right)\right)$.

Moreover, let $\left\{e_{j}\right\}_{j=1 \ldots s}$ (respectively, $\left\{f_{i}\right\}_{i=1 \ldots r}$ ) be a k-basis of $\omega\left(\mathscr{L}_{2}\right)$ (respectively, $\left.\omega\left(\mathscr{L}_{1}\right)\right)$. Then

$$
\left\{\omega(i)\left(e_{i}\right), s\left(f_{j}\right)\right\}_{i=1, \ldots, s, j=1, \ldots r}
$$

is a k-basis of $\omega(\mathscr{U})$. If $g \in \operatorname{Stab}^{\delta}\left(\mathscr{L}_{1} \oplus \mathscr{L}_{2}\right) \cap \operatorname{ker}\left(\zeta_{\mathscr{U}}\right)$, then $g$ induces the identity on

$$
\left\{\omega(i)\left(e_{i}\right), s\left(f_{j}\right)\right\}_{i=1, \ldots, s, j=1, \ldots r}
$$


and thereby on $\omega(\mathscr{U})$. Therefore, by definition of $\mathrm{Gal}^{\delta}(\mathscr{U})$, the element $g$ is the identity element and, therefore, $\operatorname{ker}\left(\left.\zeta_{\mathscr{U}}\right|_{\operatorname{Stab}^{\delta}\left(\mathscr{L}_{1} \oplus \mathscr{L}_{2}\right)}\right)$ is trivial.

Since $\operatorname{Hom}\left(\omega\left(\mathscr{L}_{1}\right), \omega\left(\mathscr{L}_{2}\right)\right)$ is abelian, the same holds for $\operatorname{Stab}^{\delta}\left(\mathscr{L}_{1} \oplus \mathscr{L}_{2}\right)$. Moreover, $\operatorname{Stab}^{\delta}\left(\mathscr{L}_{1} \oplus \mathscr{L}_{2}\right)$ is unipotent. Indeed, let $e$ be the identity element in $\operatorname{Gal}^{\delta}(\mathscr{U})$, $x \in \omega\left(\mathscr{L}_{1}\right)$, and $g \in \operatorname{Stab}^{\delta}\left(\mathscr{L}_{1} \oplus \mathscr{L}_{2}\right)$. Since $g s(x)-s(x) \in \omega\left(\mathscr{L}_{2}\right)$, we have

$$
(g-e)^{2}(s(x))=(g-e)(g s(x)-s(x))=g(g s(x)-s(x))-(g s(x)-s(x))=0 .
$$

Reasoning as above, we find that $(g-e)^{2}$ is zero on $\omega(\mathscr{U})$. By Lemma 3.2, $\operatorname{Stab}^{\delta}\left(\mathscr{L}_{1} \oplus\right.$ $\left.\mathscr{L}_{2}\right)$ is also normal and, hence, must be contained in $R_{u}\left(\mathrm{Gal}^{\delta}(\mathscr{U})\right)$. By [10, Theorem 1], the image of a unipotent linear differential algebraic group is unipotent. By Lemma $3.5, \operatorname{Stab}^{\delta}\left(\mathscr{L}_{1} \oplus \mathscr{L}_{2}\right)$ is the kernel of the projection of $\mathrm{Gal}^{\delta}(\mathscr{U})$ on the reductive linear differential algebraic group $\mathrm{Gal}^{\delta}\left(\mathscr{L}_{1} \oplus \mathscr{L}_{2}\right)$. It follows that $R_{u}\left(\mathrm{Gal}^{\delta}(\mathscr{U})\right)$ is contained in $\operatorname{Stab}^{\delta}\left(\mathscr{L}_{1} \oplus \mathscr{L}_{2}\right)$, which ends the proof.

Remark 3.7 Since two sections of (3.1) differ by a map from $\omega\left(\mathscr{L}_{1}\right)$ to $\omega\left(\mathscr{L}_{2}\right)$, one sees that, when restricted to $R_{u}\left(\mathrm{Gal}^{\delta}(\mathscr{U})\right)=\operatorname{Stab}^{\delta}\left(\mathscr{L}_{1} \oplus \mathscr{L}_{2}\right)$, the map $\zeta_{\mathscr{U}}$ is independent of the choice of the section.

By the above lemma, $R_{u}\left(\mathrm{Gal}^{\delta}(\mathscr{U})\right)$ is an abelian normal subgroup of $\mathrm{Gal}^{\delta}(\mathscr{U})$. Since $\mathrm{Gal}^{\delta}\left(\mathscr{L}_{1} \oplus \mathscr{L}_{2}\right)$ is the quotient of $\mathrm{Gal}^{\delta}(\mathscr{U})$ by $R_{u}\left(\mathrm{Gal}^{\delta}(\mathscr{U})\right)$ and $R_{u}\left(\mathrm{Gal}^{\delta}(\mathscr{U})\right)$ is abelian, the linear differential algebraic group $\mathrm{Gal}^{\delta}\left(\mathscr{L}_{1} \oplus \mathscr{L}_{2}\right)$ acts by conjugation on $R_{u}\left(\mathrm{Gal}^{\delta}(\mathscr{U})\right)$. The lemma below shows that this action is compatible with the action of $\mathrm{Gal}^{\delta}\left(\mathscr{L}_{1} \oplus \mathscr{L}_{2}\right)$ on $\operatorname{Hom}_{\mathbf{k}}\left(\omega\left(\mathscr{L}_{1}\right), \omega\left(\mathscr{L}_{2}\right)\right)$.

Lemma 3.8 For all $g_{1} \in \operatorname{Gal}^{\delta}(\mathscr{U}), g_{2} \in R_{u}\left(\mathrm{Gal}^{\delta}(\mathscr{U})\right)$, and $x \in \omega\left(\mathscr{L}_{1}\right)$, we have

$$
\zeta_{\mathscr{U}}\left(g_{1} g_{2} g_{1}^{-1}\right)(x)=g_{1}\left(\zeta_{\mathscr{U}}\left(g_{2}\right)\left(g_{1}^{-1} x\right)\right)=g_{1} * \zeta_{\mathscr{U}}\left(g_{2}\right)(x),
$$

where $*$ denotes the natural action of $\mathrm{Gal}^{\delta}\left(\mathscr{L}_{1} \oplus \mathscr{L}_{2}\right)$ on $\operatorname{Hom}\left(\omega\left(\mathscr{L}_{1}\right), \omega\left(\mathscr{L}_{2}\right)\right)$ via

$$
g * \phi=g \circ \phi \circ g^{-1} \text { for } \phi \in \operatorname{Hom}\left(\omega\left(\mathscr{L}_{1}\right), \omega\left(\mathscr{L}_{2}\right)\right) \text { and } g \in \mathrm{Gal}^{\delta}\left(\mathscr{L}_{1} \oplus \mathscr{L}_{2}\right) .
$$

Proof Let $e$ denote the identity element in $\mathrm{Gal}^{\delta}(\mathscr{U})$. From (3.2), we find that, for all $x \in \omega\left(\mathscr{L}_{1}\right)$,

$$
g_{1} \zeta_{\mathscr{U}}\left(g_{1}^{-1}\right)\left(g_{1}^{-1} x\right)=\zeta_{\mathscr{U}}(e)(x)-\zeta_{\mathscr{U}}\left(g_{1}\right)(x)=-\zeta_{\mathscr{U}}\left(g_{1}\right)(x) .
$$

Applying repeatedly (3.2), we deduce that

$$
\begin{aligned}
\zeta_{\mathscr{U}} & \left(g_{1} g_{2} g_{1}^{-1}\right)(x)=g_{1}\left(\zeta_{\mathscr{U}}\left(g_{2} g_{1}^{-1}\right)\left(g_{1}^{-1} x\right)\right)+\zeta_{\mathscr{U}}\left(g_{1}\right)(x) \\
& =g_{1}\left(g_{2} \zeta_{\mathscr{U}}\left(g_{1}^{-1}\right)\left(g_{2}^{-1} g_{1}^{-1} x\right)+\zeta_{\mathscr{U}}\left(g_{2}\right)\left(g_{1}^{-1} x\right)\right)+\zeta_{\mathscr{U}}\left(g_{1}\right)(x) \\
& =g_{1} \zeta_{\mathscr{U}}\left(g_{2}\right)\left(g_{1}^{-1} x\right)+g_{1} g_{2} g_{1}^{-1}\left(g_{1} \zeta_{\mathscr{U}}\left(g_{1}^{-1}\right)\left(g_{1}^{-1} g_{1} g_{2}^{-1} g_{1}^{-1} x\right)\right)+\zeta_{\mathscr{U}}\left(g_{1}\right)(x),
\end{aligned}
$$

for all $x \in \omega\left(\mathscr{L}_{1}\right)$. Since

$$
g_{1} g_{2} g_{1}^{-1}, g_{1} g_{2}^{-1} g_{1}^{-1} \in R_{u}\left(\operatorname{Gal}^{\delta}(\mathscr{U})\right)=\operatorname{Stab}^{\delta}\left(\mathscr{L}_{1} \oplus \mathscr{L}_{2}\right),
$$


we get that, for all $x \in \omega\left(\mathscr{L}_{1}\right)$,

$$
\begin{aligned}
g_{1} g_{2} g_{1}^{-1} & \left(g_{1} \zeta_{\mathscr{U}}\left(g_{1}^{-1}\right)\left(g_{1}^{-1} g_{1} g_{2}^{-1} g_{1}^{-1} x\right)\right)+\zeta_{\mathscr{U}}\left(g_{1}\right)(x) \\
& =g_{1} \zeta_{\mathscr{U}}\left(g_{1}^{-1}\right)\left(g_{1}^{-1} x\right)+\zeta_{\mathscr{U}}\left(g_{1}\right)(x)=0 .
\end{aligned}
$$

We conclude that, for all $x \in \omega\left(\mathscr{L}_{1}\right)$,

$$
\zeta_{\mathscr{U}}\left(g_{1} g_{2} g_{1}^{-1}\right)(x)=g_{1} \zeta_{\mathscr{U}}\left(g_{2}\right)\left(g_{1}^{-1} x\right)
$$

Proof (Proof of Theorem 3.3) By the above, $\mathrm{Gal}^{\delta}(\mathscr{U})$ is an extension of $\mathrm{Gal}^{\delta}\left(\mathscr{L}_{1} \oplus\right.$ $\left.\mathscr{L}_{2}\right)$ by $R_{u}\left(\mathrm{Gal}^{\delta}(\mathscr{U})\right)$. The action of $\mathrm{Gal}^{\delta}\left(\mathscr{L}_{1} \oplus \mathscr{L}_{2}\right)$ on $R_{u}\left(\mathrm{Gal}^{\delta}(\mathscr{U})\right)$ is deduced from the action by conjugation of $\mathrm{Gal}^{\delta}(\mathscr{U})$ on its unipotent radical.

Combining Lemma 3.6 and Lemma 3.8, we can identify via $\zeta_{\mathscr{U}}$, the unipotent radical $R_{u}\left(\operatorname{Gal}^{\delta}(\mathscr{U})\right)$ with a $\delta$-closed subgroup of $\operatorname{Hom}\left(\omega\left(\mathscr{L}_{1}\right), \omega\left(\mathscr{L}_{2}\right)\right)$ and the action of $\mathrm{Gal}^{\delta}\left(\mathscr{L}_{1} \oplus \mathscr{L}_{2}\right)$ on $R_{u}\left(\mathrm{Gal}^{\delta}(\mathscr{U})\right)$ by conjugation with the action of $\mathrm{Gal}^{\delta}\left(\mathscr{L}_{1} \oplus \mathscr{L}_{2}\right)$ on $\operatorname{Hom}\left(\omega\left(\mathscr{L}_{1}\right), \omega\left(\mathscr{L}_{2}\right)\right)$, induced by the $\operatorname{Gal}^{\delta}\left(\mathscr{L}_{1} \oplus \mathscr{L}_{2}\right)$-module structure on $\omega\left(\mathscr{L}_{1} \oplus\right.$ $\left.\mathscr{L}_{2}\right)$.

Remark 3.9 The extension in Theorem 3.3 does not split in general. For example,

$$
G=\left\{\left(\begin{array}{lll}
a & 0 & 0 \\
0 & 1 & b \\
0 & 0 & 1
\end{array}\right) \in \mathrm{GL}_{3}(\mathbf{k}) \mid \delta(b)=\frac{\delta(a)}{a}\right\}
$$

is a linear differential algebraic group such that the quotient map $G \rightarrow G / R_{u}(G) \cong \mathbf{k}^{\times}$ does not have any $\delta$-polynomial section. Indeed, otherwise, $G$ would have a projection onto $R_{u}(G) \cong C=\mathbf{k}^{\delta}$, which is impossible, because $G$ is strongly connected [12, Example 2.25].

Remark 3.10 If $K=\mathbf{k}(x)$ and $\partial=\frac{\partial}{\partial x}$, the knowledge of $R=R_{u}\left(\mathrm{Gal}^{\delta}(\mathscr{U})\right)$ allows one to compute $G=\operatorname{Gal}^{\delta}(\mathscr{U})$ algorithmically. Indeed, one can compute the normalizer $N$ of $R$ in $\operatorname{GL}(\omega(\mathscr{U}))$. Note that $G \subset N$. By the differential version of the Chevalley theorem [33, Theorem 5.1] (see also [6, proof of Theorem 5.6]), there is $\mathscr{U}_{0} \in\{\mathscr{U}\}^{\otimes, \delta}$ and a differential representation $\rho: N \rightarrow \mathrm{GL}\left(\omega\left(\mathscr{U}_{0}\right)\right)$ such that $R=\operatorname{ker} \rho$. The proof of this Chevalley theorem leads to a constructive procedure to find $\mathscr{U}_{0}$ and $\rho$. Since $\operatorname{Gal}^{\delta}\left(\mathscr{U}_{0}\right)=\rho(G)$ is reductive, one can compute it [36]. We can find $G$ as $\rho^{-1}\left(\mathrm{Gal}^{\delta}\left(\mathscr{U}_{0}\right)\right)$.

In view of Remark 3.10, our aim is to compute the parameterized differential Galois group of $\mathscr{U}$. To this purpose, we will perform a first reduction that will allow us to simplify our computation. 


\subsection{A first reduction}

Let $L_{1}, L_{2} \in K[\partial]$ be two completely reducible $\partial$-operators. Let us denote the $\partial$-module over $K$ corresponding to $L_{1}(y)=0$ (respectively, $L_{2}(y)=0$ ) by $\mathscr{L}_{1}$ (respectively, by $\mathscr{L}_{2}$ ). The $\partial$-module $\mathscr{U}$ corresponding to $L_{1}\left(L_{2}(y)\right)=0$ is an extension of $\mathscr{L}_{1}$ by $\mathscr{L}_{2}$,

$$
0 \longrightarrow \mathscr{L}_{2} \stackrel{i}{\longrightarrow} \mathscr{U} \stackrel{p}{\longrightarrow} \mathscr{L}_{1} \longrightarrow 0
$$

in the category of $\partial$-modules over $K$. In this section, we recall the methods of [4] to show that we can restrict ourselves to the case in which $L_{1}$ is of the form $\partial-\frac{\partial b}{b}$ for some $b \in K^{*}$.

We first describe the reduction process in terms of $\partial$-modules. Since the functor $\underline{\operatorname{Hom}}\left(\mathscr{L}_{1},-\right)$ is exact, (3.4) gives the exact sequence:

$$
0 \longrightarrow \underline{\operatorname{Hom}}\left(\mathscr{L}_{1}, \mathscr{L}_{2}\right) \longrightarrow \underline{\operatorname{Hom}}\left(\mathscr{L}_{1}, \mathscr{U}\right) \longrightarrow \underline{\operatorname{Hom}}\left(\mathscr{L}_{1}, \mathscr{L}_{1}\right) \longrightarrow 0
$$

We pull back (3.5) by the diagonal embedding

$$
d: \mathbf{1} \rightarrow \underline{\operatorname{Hom}}\left(\mathscr{L}_{1}, \mathscr{L}_{1}\right), \quad \lambda \mapsto \lambda \operatorname{id}_{\mathscr{L}_{1}},
$$

where $\mathbf{1}$ is the unit object. We obtain an exact sequence

$$
0 \longrightarrow \underline{\operatorname{Hom}}\left(\mathscr{L}_{1}, \mathscr{L}_{2}\right) \longrightarrow \mathscr{R}(\mathscr{U}) \longrightarrow 1 \longrightarrow 0
$$

where $\mathscr{R}(\mathscr{U})$ is the $\partial$-module deduced from $\mathscr{U}$ by the pullback. We call the $\partial$-module $\mathscr{R}(\mathscr{U})$ the reduction of $\mathscr{U}$. We recall that, as a $K$-vector space, $\mathscr{R}(\mathscr{U})$ coincides with the set

$$
\left\{(\phi, \lambda) \in \underline{\operatorname{Hom}}\left(\mathscr{L}_{1}, \mathscr{U}\right) \times \mathbf{1} \mid p \circ \phi=\lambda \operatorname{id}_{\mathscr{L}_{1}}\right\}
$$

Remark 3.11 An effective interpretation of this reduction process in terms of matrix differential equations immediately follows from [4, page 15].

Proposition 3.12 With notation above, we have

(1) The parameterized differential Galois group $\mathrm{Gal}^{\delta}\left(\underline{\operatorname{Hom}}\left(\mathscr{L}_{1}, \mathscr{L}_{2}\right)\right)$ is a quotient of $\mathrm{Gal}^{\delta}\left(\mathscr{L}_{1} \oplus \mathscr{L}_{2}\right)$ and is a reductive linear differential algebraic group;

(2) By Lemma 3.6, one can identify $R_{u}\left(\mathrm{Gal}^{\delta}(\mathscr{U})\right.$ ) (respectively, $R_{u}\left(\mathrm{Gal}^{\delta}(\mathscr{R}(\mathscr{U}))\right)$ ) with a differential algebraic subgroup of $\operatorname{Hom}\left(\omega\left(\mathscr{L}_{1}\right), \omega\left(\mathscr{L}_{2}\right)\right.$ ) (respectively, of $\left.\operatorname{Hom}\left(\mathbf{k}, \operatorname{Hom}\left(\omega\left(\mathscr{L}_{1}\right), \omega\left(\mathscr{L}_{2}\right)\right)\right)\right)$. Then the canonical isomorphism

$$
\phi: \operatorname{Hom}\left(\mathbf{k}, \operatorname{Hom}\left(\omega\left(\mathscr{L}_{1}\right), \omega\left(\mathscr{L}_{2}\right)\right)\right) \rightarrow \operatorname{Hom}\left(\omega\left(\mathscr{L}_{1}\right), \omega\left(\mathscr{L}_{2}\right)\right), \psi \mapsto \psi(1)
$$

induces an isomorphism of linear differential algebraic groups between $R_{u}\left(\mathrm{Gal}^{\delta}(\mathscr{R}(\mathscr{U}))\right)$ and $R_{u}\left(\mathrm{Gal}^{\delta}(\mathscr{U})\right)$;

(3) By Lemma 3.8, $\operatorname{Gal}^{\delta}\left(\mathscr{L}_{1} \oplus \mathscr{L}_{2}\right)$ (respectively, $\operatorname{Gal}^{\delta}\left(\underline{\operatorname{Hom}}\left(\mathscr{L}_{1}, \mathscr{L}_{2}\right)\right)$ ) acts on $R_{u}\left(\mathrm{Gal}^{\delta}(\mathscr{U})\right)$ (respectively, on $R_{u}\left(\mathrm{Gal}^{\delta}(\mathscr{R}(\mathscr{U}))\right)$ ). These actions are compatible with the isomorphism $\phi$. 


\section{Proof}

(1) Since $\underline{\operatorname{Hom}}\left(\mathscr{L}_{1}, \mathscr{L}_{2}\right)$ (respectively, $\left.\mathscr{L}_{1} \oplus \mathscr{L}_{2}\right)$ is a subobject of $\{\mathscr{U}\}^{\otimes, \delta}$, its parameterized differential Galois group is a quotient of $\operatorname{Gal}^{\delta}(\mathscr{U})$ by $\operatorname{Stab}^{\delta}\left(\underline{\operatorname{Hom}}\left(\mathscr{L}_{1}, \mathscr{L}_{2}\right)\right)$ (respectively, by $\left.\operatorname{Stab}^{\delta}\left(\mathscr{L}_{1} \oplus \mathscr{L}_{2}\right)=\operatorname{Stab}^{\delta}\left(\mathscr{L}_{1}\right) \cap \operatorname{Stab}^{\delta}\left(\mathscr{L}_{2}\right)\right)$. It is not difficult to see that we have the inclusion

$$
\operatorname{Stab}^{\delta}\left(\mathscr{L}_{1} \oplus \mathscr{L}_{2}\right) \subset \operatorname{Stab}^{\delta}\left(\underline{\operatorname{Hom}}\left(\mathscr{L}_{1}, \mathscr{L}_{2}\right)\right)
$$

Since stabilizers of objects in $\{\mathscr{U}\}^{\otimes, \delta}$ are normal in $\mathrm{Gal}^{\delta}(\mathscr{U})$ by Lemma 3.2, we can apply [10, Proposition 2] to get that

$$
\operatorname{Gal}^{\delta}\left(\underline{\operatorname{Hom}}\left(\mathscr{L}_{1}, \mathscr{L}_{2}\right)\right)=\operatorname{Gal}^{\delta}(\mathscr{U}) / \operatorname{Stab}^{\delta}\left(\underline{\operatorname{Hom}}\left(\mathscr{L}_{1}, \mathscr{L}_{2}\right)\right)
$$

is a quotient of

$$
\operatorname{Gal}^{\delta}\left(\mathscr{L}_{1} \oplus \mathscr{L}_{2}\right)=\operatorname{Gal}^{\delta}(\mathscr{U}) / \operatorname{Stab}^{\delta}\left(\mathscr{L}_{1} \oplus \mathscr{L}_{2}\right)
$$

by

$$
\operatorname{Stab}^{\delta}\left(\underline{\operatorname{Hom}}\left(\mathscr{L}_{1}, \mathscr{L}_{2}\right)\right) / \operatorname{Stab}^{\delta}\left(\mathscr{L}_{1} \oplus \mathscr{L}_{2}\right)
$$

The same reasoning in the non-parameterized case shows that $\operatorname{Gal}\left(\underline{\operatorname{Hom}}\left(\mathscr{L}_{1}, \mathscr{L}_{2}\right)\right)$ is a quotient of $\operatorname{Gal}\left(\mathscr{L}_{1} \oplus \mathscr{L}_{2}\right)$. Since quotients of reductive algebraic groups are reductive, [36, Remark 2.9] allows us to conclude that $\operatorname{Gal}^{\delta}\left(\underline{\operatorname{Hom}}\left(\mathscr{L}_{1}, \mathscr{L}_{2}\right)\right)$ is a reductive linear differential algebraic group.

(2) Since $\mathscr{R}(\mathscr{U})$ is an object of $\{\mathscr{U}\}^{\otimes, \delta}, \mathrm{Gal}^{\delta}(\mathscr{R}(\mathscr{U}))$ is a quotient of $\mathrm{Gal}^{\delta}(\mathscr{U})$, and we denote the canonical surjection by $\pi$. The image of $\operatorname{Stab} \delta\left(\underline{\operatorname{Hom}}\left(\mathscr{L}_{1}, \mathscr{L}_{2}\right)\right)$ via $\pi$ coincides with the stabilizer of $\underline{\operatorname{Hom}}\left(\mathscr{L}_{1}, \mathscr{L}_{2}\right)$ in $\operatorname{Gal}^{\delta}(\mathscr{R}(\mathscr{\mathscr { U }}))$ and, thus, with $R_{u}\left(\mathrm{Gal}^{\delta}(\mathscr{R}(\mathscr{U}))\right)$ by Lemmas 3.5 and 3.6.

Let $H \subset R_{u}\left(\mathrm{Gal}^{\delta}(\mathscr{R}(\mathscr{U}))\right)$ be the image of $\operatorname{Stab}^{\delta}\left(\mathscr{L}_{1} \oplus \mathscr{L}_{2}\right)$ by $\pi$. By [8, Proposition 7, page 908], $H$ is a differential algebraic subgroup of $R_{u}\left(\mathrm{Gal}^{\delta}(\mathscr{R}(\mathscr{U}))\right)$. Since $\operatorname{Stab}^{\delta}\left(\mathscr{L}_{1} \oplus \mathscr{L}_{2}\right)$ is normal in $\operatorname{Gal}^{\delta}(\mathscr{U})$ and $\pi$ is surjective, $H$ is normal in $R_{u}\left(\mathrm{Gal}^{\delta}(\mathscr{R}(\mathscr{U}))\right)$, and we can consider the quotient map

$$
p: R_{u}\left(\operatorname{Gal}^{\delta}(\mathscr{R}(\mathscr{U}))\right) \rightarrow R_{u}\left(\mathrm{Gal}^{\delta}(\mathscr{R}(\mathscr{U}))\right) / H .
$$

Since quotients of unipotent linear differential algebraic groups are unipotent by [10, Theorem 1], the linear differential algebraic group $R_{u}\left(\mathrm{Gal}^{\delta}(\mathscr{R}(\mathscr{U}))\right) / H$ is unipotent. Note that

$$
R_{u}\left(\operatorname{Gal}^{\delta}(\mathscr{R}(\mathscr{U}))\right) / H=\pi\left(\operatorname{Stab}^{\delta}\left(\underline{\operatorname{Hom}}\left(\mathscr{L}_{1}, \mathscr{L}_{2}\right)\right)\right) / \pi\left(\operatorname{Stab}^{\delta}\left(\mathscr{L}_{1} \oplus \mathscr{L}_{2}\right)\right)
$$

The surjective morphism $\pi$ is induced via $\delta$-Tannakian equivalence by the inclusion of $\delta$-Tannakian categories $\{\mathscr{R}(\mathscr{U})\}^{\otimes, \delta} \subset\{\mathscr{U}\}^{\otimes, \delta}$. This inclusion restricts to the inclusion of the usual Tannakian categories $\{\mathscr{R}(\mathscr{U})\}^{\otimes} \subset\{\mathscr{U}\}^{\otimes}$, which shows, taking the Zariski closure, that $\pi$ extends to a surjective morphism of algebraic groups $\bar{\pi}: \operatorname{Gal}(\mathscr{U}) \rightarrow \operatorname{Gal}(\mathscr{R}(\mathscr{U}))$. One can show that the quotient

$$
\bar{\pi}\left(\operatorname{Stab}\left(\underline{\operatorname{Hom}}\left(\mathscr{L}_{1}, \mathscr{L}_{2}\right)\right)\right) / \bar{\pi}\left(\operatorname{Stab}\left(\mathscr{L}_{1} \oplus \mathscr{L}_{2}\right)\right)
$$


coincides with the Zariski closure of $R_{u}\left(\mathrm{Gal}^{\delta}(\mathscr{R}(\mathscr{U}))\right) / H$.

Let $K_{\mathscr{L}_{1} \oplus \mathscr{L}_{2}}$ (respectively, $K_{\underline{\text { Hom }}}\left(\mathscr{L}_{1}, \mathscr{L}_{2}\right)$ ) denote the usual PV extension of $\mathscr{L}_{1} \oplus \mathscr{L}_{2}$ (respectively, of $\underline{\operatorname{Hom}}\left(\mathscr{L}_{1}, \overline{\mathscr{L}}_{2}\right)$ ) over $K$. Let $K_{\mathscr{U}}$ (respectively, $K_{R(\mathscr{U})}$ ) denote the usual PV extension of $\mathscr{U}$ (respectively, of $\mathscr{R}(\mathscr{U}))$ ) over $K$. We have the following tower of $\partial$-field extensions:

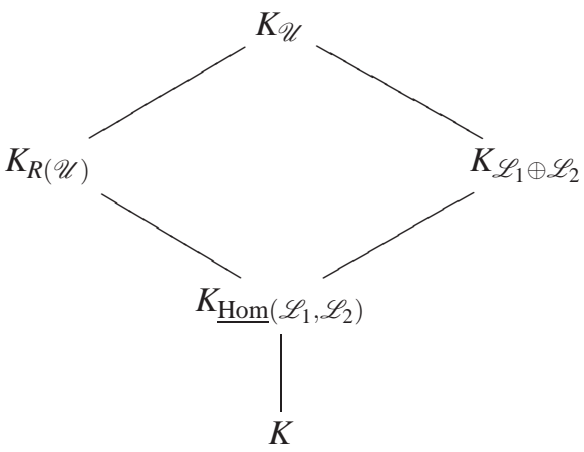

We see that

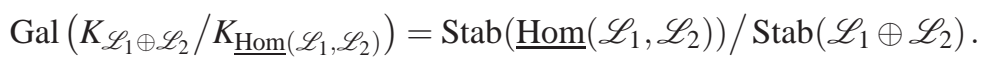

Since $K_{\underline{\operatorname{Hom}}}\left(\mathscr{L}_{1}, \mathscr{L}_{2}\right)$ is a PV extension of $K$, the group $\operatorname{Gal}\left(K_{\mathscr{L}_{1} \oplus \mathscr{L}_{2}} / K_{\underline{\operatorname{Hom}}}\left(\mathscr{L}_{1}, \mathscr{L}_{2}\right)\right)$ is normal

in

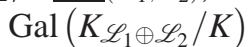

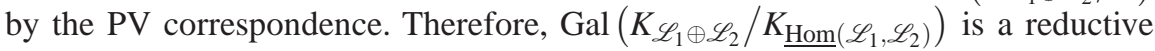
algebraic group. Since

$$
\begin{aligned}
\left.\bar{\pi}: \operatorname{Stab}\left(\underline{\operatorname{Hom}}\left(\mathscr{L}_{1}, \mathscr{L}_{2}\right)\right) / \operatorname{Stab}\left(\mathscr{L}_{1} \oplus \mathscr{L}_{2}\right)\right) \\
\rightarrow \bar{\pi}\left(\operatorname{Stab}\left(\underline{\operatorname{Hom}}\left(\mathscr{L}_{1}, \mathscr{L}_{2}\right)\right)\right) / \bar{\pi}\left(\operatorname{Stab}\left(\mathscr{L}_{1} \oplus \mathscr{L}_{2}\right)\right)
\end{aligned}
$$

is a quotient map, we deduce from the above identifications that the Zariski closure of $R_{u}\left(\mathrm{Gal}^{\delta}(\mathscr{R}(\mathscr{U}))\right) / H$ is a reductive algebraic group. We conclude by [36, Remark 2.9] that $R_{u}\left(\mathrm{Gal}^{\delta}(\mathscr{R}(\mathscr{U}))\right) / H$ is reductive. On the other hand, since $R_{u}\left(\operatorname{Gal}^{\delta}(\mathscr{R}(\mathscr{U}))\right) / H$ is both unipotent and reductive, it must be equal to $\{e\}$, and we have

$$
\pi\left(\operatorname{Stab}^{\delta}\left(\mathscr{L}_{1} \oplus \mathscr{L}_{2}\right)\right)=\pi\left(\operatorname{Stab}^{\delta}\left(\underline{\operatorname{Hom}}\left(\mathscr{L}_{1}, \mathscr{L}_{2}\right)\right)\right)=R_{u}\left(\operatorname{Gal}^{\delta}(\mathscr{R}(\mathscr{U}))\right) .
$$

We recall the notation of Lemma 3.6. We denote by $s$ a k-linear section of the exact sequence of finite-dimensional representations of $\mathrm{Gal}^{\delta}(\mathscr{U})$ :

$$
0 \longrightarrow \omega\left(\mathscr{L}_{2}\right) \stackrel{\omega(i)}{\longrightarrow} \omega(\mathscr{U}) \underset{\text { భ.... }}{\stackrel{\omega(p)}{\longrightarrow}} \omega\left(\mathscr{L}_{1}\right) \longrightarrow 0
$$

Then, we identify $R_{u}\left(\operatorname{Gal}^{\delta}(\mathscr{U})\right)=\operatorname{Stab}^{\delta}\left(\mathscr{L}_{1} \oplus \mathscr{L}_{2}\right)$ with the image of $\operatorname{Stab}^{\delta}\left(\mathscr{L}_{1} \oplus\right.$ $\left.\mathscr{L}_{2}\right)$ by

$$
\zeta_{\mathscr{U}}: R_{u}\left(\operatorname{Gal}^{\delta}(\mathscr{U})\right) \rightarrow \operatorname{Hom}_{\mathbf{k}}\left(\omega\left(\mathscr{L}_{1}\right), \omega\left(\mathscr{L}_{2}\right)\right), \quad g \mapsto\left(x \mapsto g s\left(g^{-1} x\right)-s(x)\right) .
$$


Since $\omega$ is compatible with $\underline{\text { Hom}}$, the map

$$
r: \mathbf{k} \rightarrow \omega(\mathscr{R}(\mathscr{U})), \quad \lambda \mapsto(\lambda s, \lambda),
$$

is a $\mathbf{k}$-linear section of $t$

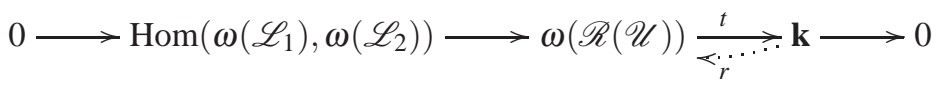

We apply again Lemma 3.6 to identify $R_{u}\left(\operatorname{Gal}^{\delta}(\mathscr{R}(\mathscr{U}))\right)=\pi\left(\operatorname{Stab}^{\delta}\left(\mathscr{L}_{1} \oplus \mathscr{L}_{2}\right)\right)$ with its image via

$$
\begin{aligned}
\zeta_{\mathscr{R}(\mathscr{U})}: \operatorname{Gal}^{\delta}(\mathscr{R}(\mathscr{U})) & \rightarrow \operatorname{Hom}\left(\mathbf{k}, \operatorname{Hom}_{\mathbf{k}}\left(\omega\left(\mathscr{L}_{1}\right), \omega\left(\mathscr{L}_{2}\right)\right)\right) \\
g & \mapsto\left(\lambda \mapsto g r(\lambda) g^{-1}-r(\lambda)\right) .
\end{aligned}
$$

Identifying $\operatorname{Hom}\left(\mathbf{k}, \operatorname{Hom}\left(\omega\left(\mathscr{L}_{1}\right), \omega\left(\mathscr{L}_{2}\right)\right)\right)$ with $\operatorname{Hom}\left(\omega\left(\mathscr{L}_{1}\right), \omega\left(\mathscr{L}_{2}\right)\right)$ via $\phi$, we find that

$$
\zeta_{\mathscr{U}}=\phi \circ \zeta_{\mathscr{R}(\mathscr{U})} \circ \pi
$$

We have

$$
\begin{aligned}
R_{u}\left(\operatorname{Gal}^{\delta}(\mathscr{U})\right) & =\zeta_{\mathscr{U}}\left(\operatorname{Stab}^{\delta}\left(\mathscr{L}_{1} \oplus \mathscr{L}_{2}\right)\right) \\
& =\zeta_{\mathscr{R}(\mathscr{U})} \circ \pi\left(\operatorname{Stab}^{\delta}\left(\mathscr{L}_{1} \oplus \mathscr{L}_{2}\right)\right)=R_{u}\left(\operatorname{Gal}^{\delta}(\mathscr{R}(\mathscr{U}))\right),
\end{aligned}
$$

where we have used Remark 3.7.

(3) The compatibility of the actions comes from Lemma 3.8, (3.9), and (3.8).

We combine Proposition 3.12 and Theorem 3.3 in the following Theorem.

Theorem 3.13 If $\mathscr{L}_{1}, \mathscr{L}_{2}$ are completely reducible $\partial$-modules over $K$ and if $\mathscr{U}$ is a $\partial$-module extension of $\mathscr{L}_{1}$ by $\mathscr{L}_{2}$, then

(1) $\mathrm{Gal}^{\delta}(\mathscr{U})$ is an extension of $\mathrm{Gal}^{\delta}\left(\mathscr{L}_{1} \oplus \mathscr{L}_{2}\right)$ by a $\delta$-subgroup $W$ of $\omega\left(\underline{\operatorname{Hom}}\left(\mathscr{L}_{1}, \mathscr{L}_{2}\right)\right)$.

(2) $W=R_{u}\left(\mathrm{Gal}^{\delta}(\mathscr{R}(\mathscr{U}))\right)$, where $\mathscr{R}(\mathscr{U})$ is an extension of $\mathbf{1}$ by the completely reducible $\partial$-module $\underline{\operatorname{Hom}}\left(\mathscr{L}_{1}, \mathscr{L}_{2}\right)$, and the action of $\mathrm{Gal}^{\delta}\left(\mathscr{L}_{1} \oplus \mathscr{L}_{2}\right)$ on $W$ is given by composing the quotient map of $\mathrm{Gal}^{\delta}\left(\mathscr{L}_{1} \oplus \mathscr{L}_{2}\right)$ on $\mathrm{Gal}^{\delta}\left(\underline{\operatorname{Hom}}\left(\mathscr{L}_{1}, \mathscr{L}_{2}\right)\right)$ with the action of $\mathrm{Gal}^{\delta}\left(\underline{\operatorname{Hom}}\left(\mathscr{L}_{1}, \mathscr{L}_{2}\right)\right)$ on $\omega\left(\underline{\operatorname{Hom}}\left(\mathscr{L}_{1}, \mathscr{L}_{2}\right)\right)$.

3.3 The unipotent radical of the parameterized differential Galois group of an extension of 1 by a completely reducible $\partial$-module $\mathscr{L}$

Let $\mathscr{L}$ be a completely reducible $\partial$-module over $K$ and $\mathscr{U}$ be an extension of $\mathbf{1}$ by $\mathscr{L}$. In this section, we study $R_{u}\left(\mathrm{Gal}^{\delta}(\mathscr{U})\right)$.

In terms of $\partial$-operators, the situation corresponds to the following. Let $L \in K[\partial]$ be a completely reducible $\partial$-operator and $\mathscr{L}$ be the associated $\partial$-module. An extension $\mathscr{U}$ of 1 by $\mathscr{L}$ corresponds to an inhomogeneous differential equation of the form $L(y)=b$ for some $b \in K^{*}$. The main result of [4] is to show that $R_{u}(\operatorname{Gal}(\mathscr{U}))=\omega\left(\mathscr{L}_{0}\right)$, where $\mathscr{L}_{0}$ is the largest $\partial$-module of $\mathscr{L}$ such that 
(1) $L=L_{1} L_{0}$;

(2) $L_{1}(y)=b$ has a solution in $K$.

From Lemma 3.6, we know that $R_{u}\left(\mathrm{Gal}^{\delta}(\mathscr{U})\right)$ can be identified with a differential algebraic subgroup $W$ of $\omega\left(\mathscr{L}_{0}\right)$, stable under the natural action of $\mathrm{Gal}^{\delta}(\mathscr{L})$ on $\omega(\mathscr{L})$. In [21], the result of [4] was rephrased in Tannakian terms and it was proved that $\mathscr{L}_{0}$ is the smallest subobject of $\mathscr{L}$ such that the pushout of the extension $\mathscr{U}$ by the quotient map $\pi: \mathscr{L} \rightarrow \mathscr{L} / \mathscr{L}_{0}$ is a trivial (split) extension. Such a characterization no longer holds in general in the parameterized setting. Indeed, the classification of differential algebraic subgroups of vector groups shows that $W$ coincides with the zero set of a finite system of linear homogeneous differential equations with coefficients in $\mathbf{k}$. Therefore, we have two possibilities:

- either $W$ is given by linear homogeneous polynomials and it is a finite-dimensional vector space over $\mathbf{k}$, that is, $W$ is an algebraic subgroup of $\omega\left(\mathscr{L}_{0}\right)$;

- or $W$ is given by linear homogeneous $\delta$-polynomials of order greater than 0 , and $W$ is a vector space over $C=\mathbf{k}^{\delta}$.

In the first case, we deduce from the $\delta$-Tannakian equivalence for the category $\{\mathscr{L}\}\}^{\otimes, \delta}$ that $W=\omega\left(\widetilde{\mathscr{L}}_{0}\right)$ for a submodule $\widetilde{\mathscr{L}}_{0}$ of $\mathscr{L}$ if and only if it is an algebraic subgroup of $\omega\left(\mathscr{L}_{0}\right)$. In this situation, we show that $\widetilde{\mathscr{L}}_{0}$ is the smallest $\partial$-submodule of $\mathscr{L}$ such that the parameterized differential Galois group of the pushout of the extension $\mathscr{U}$ by the quotient map $\pi: \mathscr{L} \rightarrow \mathscr{L} / \widetilde{\mathscr{L}}_{0}$ is reductive (see Theorem 3.19). This last condition can be tested by an algorithm contained in [36].

If $W$ is not given by linear homogeneous $\delta$-polynomials of order 0 , then $W$ is not of the form $\omega(\widetilde{\mathscr{L}})$ for any $\widetilde{\mathscr{L}}$. Moreover, the order of the defining equations of $W$ can be as high as required even for second order differential equations:

Example 3.14 For $n \geq 0$, let

$$
z(x, t, n)=\sum_{j=0}^{n} t^{j} \ln (x+j) ; \quad a(x, t, n)=\frac{\partial z(x, t, n)}{\partial x}=\sum_{j=0}^{n} \frac{t^{j}}{x+j} \in \mathbf{k}(x),
$$

where $\mathbf{k}$ is a differentially closed field with respect to $\partial / \partial t$ containing $\mathbb{Q}(t)$. Then the function $z(x, t, n)$ satisfies the following second order differential equation in $y(x, t)$ over $\mathbf{k}(x)$ :

$$
\frac{\partial\left(\frac{\partial y(x, t)}{\partial x} / a(x, t, n)\right)}{\partial x}=0 \quad \Longleftrightarrow \quad \frac{\partial^{2} y(x, t)}{\partial x^{2}}-\frac{\frac{\partial a(x, t, n)}{\partial x}}{a(x, t, n)} \frac{\partial y(x, t)}{\partial x}=0 .
$$

Since $\ln (x), \ldots, \ln (x+n)$ are algebraically independent over $\mathbf{k}(x)$ by $[42,16]$, and $\frac{\partial^{n+1} z(x, t, n)}{\partial t^{n+1}}=0$, and

$$
\mathbf{k}(x)(\ln (x), \ldots, \ln (x+n))=\mathbf{k}(x)\left(\frac{\partial^{j}(z(x, t, n))}{\partial t^{j}} \mid j \geq 0\right),
$$

we have

$$
\mathrm{Gal}^{\delta}=\left\{\left(\begin{array}{ll}
1 & a \\
0 & 1
\end{array}\right) \mid \frac{\partial^{n+1} a}{\partial t^{n+1}}=0\right\}
$$


In Section 3.3.1, we give a decomposition of $\mathscr{L}$ into "constant and purely nonconstant" parts, which allows us to distinguish between the two cases for the unipotent radical $W$ described above. In Section 3.3.2, we treat the "purely non-constant case". In Section 3.3.3, we give a general algorithm to compute $R_{u}\left(\mathrm{Gal}^{\delta}(\mathscr{U})\right)$ under the assumption that $\mathscr{L}$ has no non-zero trivial $\partial$-submodules in the sense of Definition 2.42 .

\subsubsection{Decomposition of the completely reducible $\partial$-module $\mathscr{L}$}

The following lemma gives a decomposition of a completely reducible $\partial$-module into a direct sum of $\partial$-modules, a "constant" one and a "purely non-constant" one.

Lemma 3.15 Let $\mathscr{L}$ be a completely reducible $\partial$-module and $\rho: \operatorname{Gal}^{\delta}(\mathscr{L}) \rightarrow$ $\mathrm{GL}(\omega(\mathscr{L}))$ be the representation of the parameterized differential Galois group of $\mathscr{L}$ on $\omega(\mathscr{L})$. Then there exist $\partial$-submodules $\mathscr{L}_{c}$ and $\mathscr{L}_{n c}$ of $\mathscr{L}$ such that

- $\mathscr{L}=\mathscr{L}_{c} \oplus \mathscr{L}_{n c}$

- the representation of $\mathrm{Gal}^{\delta}(\mathscr{L})$ on $\mathscr{L}_{c}$ is conjugate to constants in $\mathrm{GL}\left(\omega\left(\mathscr{L}_{c}\right)\right)$, that is, any differential system associated to $\mathscr{L}_{c}$ is isomonodromic by Proposition 2.52;

- $\mathscr{L}_{c}$ is maximal for the properties above, that is, there is no non-zero $\partial$-submodule $\mathscr{N}$ of $\mathscr{L}_{n c}$ such that the representation of $\mathrm{Gal}^{\delta}(\mathscr{L})$ on $\mathscr{N}$ is conjugate to constants in $\operatorname{GL}(\omega(\mathscr{N}))$.

Proof Let $\mathscr{L}_{1}, \ldots, \mathscr{L}_{r}$ be irreducible $\partial$-submodules such that $\mathscr{L}=\mathscr{L}_{1} \oplus \ldots \oplus \mathscr{L}_{r}$. We have

$$
\mathrm{GL}(\omega(\mathscr{L}))=\prod_{i=1}^{r} \mathrm{GL}\left(\omega\left(\mathscr{L}_{i}\right)\right)
$$

Let $S$ be the set of indices $i$ in $\{1, \ldots, r\}$ such that the representation of $\mathrm{Gal}^{\delta}(\mathscr{L})$ on $\omega\left(\mathscr{L}_{i}\right)$ is conjugate to constants in $\operatorname{GL}\left(\omega\left(\mathscr{L}_{i}\right)\right)$. Setting

$$
\mathscr{L}_{c}=\bigoplus_{i \in S} \mathscr{L}_{i} \quad \text { and } \quad \mathscr{L}_{n c}=\bigoplus_{i \notin S} \mathscr{L}_{i}
$$

allows to conclude the proof.

Remark 3.16 The above construction is effective. Let $\mathscr{L}$ be a completely reducible $\partial$ module over $K=\mathbb{C}(z)$ with $\partial(z)=1$ and $\partial(\mathbb{C})=0$. There are many algorithms that compute a factorization of $\mathscr{L}$ into a direct sum of irreducible $\partial$-submodules: see, for instance, $[23,48]$. Thus, we can find a linear differential system associated to $\mathscr{L}$ of the form

$$
\partial(Y)=\left(\begin{array}{cccc}
A_{1} & 0 & \ldots & 0 \\
0 & A_{2} & \ldots & 0 \\
\vdots & \ddots & \ddots & \vdots \\
0 & \ldots & 0 & A_{r}
\end{array}\right) Y
$$


with $A_{i} \in K^{n_{i} \times n_{i}}$ for all $i=1, \ldots, r$ and such that $\partial(Y)=A_{i} Y$ is an irreducible differential system. For all $i=1, \ldots, r$, let $\mathscr{L}_{i}$ be a $\partial$-module associated to $\partial(Y)=A_{i} Y$. Let $S$ be the set of indices $i$ such that there exists a matrix $B_{i} \in K^{n_{i} \times n_{i}}$ such that

$$
\delta\left(A_{i}\right)-\partial\left(B_{i}\right)=B_{i} A_{i}-A_{i} B_{i} .
$$

Since there are algorithms to find rational solutions of linear differential systems (see [3]), the construction of the set $S$ is also effective. We can set

$$
\mathscr{L}_{c}=\bigoplus_{i \in S} \mathscr{L}_{i} \quad \text { and } \quad \mathscr{L}_{n c}=\bigoplus_{i \neq S} \mathscr{L}_{i}
$$

This decomposition motivates the following definition.

Definition 3.17 A $\partial$-module $\mathscr{L}$ over $K$ is said to be constant if the representation of $\operatorname{Gal}^{\delta}(\mathscr{L})$ on $\omega(\mathscr{L})$ is conjugate to constants in $\operatorname{GL}(\omega(\mathscr{L}))$. On the contrary, the $\partial$ module $\mathscr{L}$ is said to be purely non-constant if there is no non-zero $\partial$-submodule $\mathscr{N}$ of $\mathscr{L}$ such that the representation of $\mathrm{Gal}^{\delta}(\mathscr{L})$ on $\omega(\mathscr{N})$ is conjugate to constants in $\operatorname{GL}(\omega(\mathscr{N}))$.

Remark 3.18 We say that a $G$-module $V$ is purely non-constant if, for every non-zero $G$-submodule $W$ of $V$, the induced representation $\rho: G \rightarrow \mathrm{GL}(W)$ is non-constant. By the Tannakian equivalence, a $\partial$-module $\mathscr{L}$ is purely non-constant if and only if the $\mathrm{Gal}^{\delta}(\mathscr{L})$-module $\omega(\mathscr{L})$ is purely non-constant.

Recall that $\mathscr{U}$ is a $\partial$-module extension of $\mathbf{1}$ by $\mathscr{L}$. We consider the pushout of

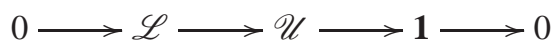

by the projection of $\mathscr{L}$ on $\mathscr{L}_{c}$ (respectively, on $\mathscr{L}_{n c}$ ). We find two exact sequences of $\partial$-modules:

$$
0 \longrightarrow \mathscr{L}_{c} \longrightarrow \mathscr{U}_{c} \longrightarrow \mathbf{1} \longrightarrow 0
$$

and

$$
0 \longrightarrow \mathscr{L}_{n c} \longrightarrow \mathscr{U}_{n c} \longrightarrow \mathbf{1} \longrightarrow 0
$$

We deduce from Lemma 3.6 that

- $R_{u}\left(\mathrm{Gal}^{\delta}(\mathscr{U})\right)$ is a differential algebraic subgroup of $\omega(\mathscr{L})$;

- $R_{u}\left(\mathrm{Gal}^{\delta}\left(\mathscr{U}_{c}\right)\right)$ is a differential algebraic subgroup of $\omega\left(\mathscr{L}_{c}\right)$;

- $R_{u}\left(\operatorname{Gal}^{\delta}\left(\mathscr{U}_{n c}\right)\right)$ is a differential algebraic subgroup of $\omega\left(\mathscr{L}_{n c}\right)$.

The quotient $\mathrm{Gal}^{\delta}\left(\mathscr{U}_{c}\right) / R_{u}\left(\mathrm{Gal}^{\delta}\left(\mathscr{U}_{c}\right)\right)$ is $\mathrm{Gal}^{\delta}\left(\mathscr{L}_{c}\right)$, which is, by construction, conjugate to constants. We can use [35] to compute $R_{u}\left(\mathrm{Gal}^{\delta}\left(\mathscr{U}_{c}\right)\right)$. Section 3.3.2 shows how to compute the unipotent radical of the parameterized differential Galois group of an extension of $\mathbf{1}$ by a purely non constant completely reducible module. Finally, Section 3.3.3 shows how to combine Section 3.3.2 with [35] to deduce $R_{u}\left(\mathrm{Gal}^{\delta}(\mathscr{U})\right)$ from the computation of $R_{u}\left(\mathrm{Gal}^{\delta}\left(\mathscr{U}_{c}\right)\right)$ and $R_{u}\left(\mathrm{Gal}^{\delta}\left(\mathscr{U}_{n c}\right)\right)$. 


\subsubsection{The purely non-constant case}

The aim of this section is to prove the following theorem.

Theorem 3.19 Let $\mathscr{L}$ be a purely non-constant completely reducible $\partial$-module over $K$. Let $\mathscr{U}$ be a $\partial$-module extension of 1 by $\mathscr{L}$. Then, $R_{u}\left(\mathrm{Gal}^{\delta}(\mathscr{U})\right)=\omega\left(\widetilde{\mathscr{L}}_{0}\right)$, where $\widetilde{\mathscr{L}}_{0}$ is the smallest $\partial$-submodule of $\mathscr{L}$ such that $\mathrm{Gal}^{\delta}\left(\mathscr{U} / \widetilde{\mathscr{L}}_{0}\right)$ is reductive.

By Theorem 3.13, $R_{u}\left(\mathrm{Gal}^{\delta}(\mathscr{U})\right)$ is a $\delta$-closed subgroup of $\omega(\mathscr{L})$, which is stable under the action of $\mathrm{Gal}^{\delta}(\mathscr{L})$. We show that any such subgroup is a $\mathbf{k}$-vector subspace. In this attempt, we first treat the cases in which $\mathrm{Gal}^{\delta}(\mathscr{L})$ is a torus or $\mathrm{SL}_{2}$. We conclude with the general situation and the proof of Theorem 3.19.

The algorithm contained in [36] allows one to test whether the unipotent radical of a linear algebraic group is trivial. This algorithm relies on bounds on the order of the defining equations of the parameterized differential Galois group. Combined with Theorem 3.19, we find a complete algorithm to compute $R_{u}\left(\mathrm{Gal}^{\delta}(\mathscr{U})\right)$.

Theorem 3.19 implies among other things that $R_{u}\left(\mathrm{Gal}^{\delta}(\mathscr{U})\right)$ is an algebraic subgroup of $R_{u}(\operatorname{Gal}(\mathscr{U}))$. Despite the fact that $\mathrm{Gal}^{\delta}(\mathscr{U})$ (respectively, $\mathrm{Gal}^{\delta}(\mathscr{L})$ ) is Zariski dense in $\operatorname{Gal}(\mathscr{U})$ (respectively, $\operatorname{Gal}(\mathscr{L})$ ), it might happen that $R_{u}\left(\mathrm{Gal}^{\delta}(\mathscr{U})\right.$ ) is contained in a proper Zariski closed subgroup of $R_{u}(\operatorname{Gal}(\mathscr{U}))$ as it is shown in the following example.

Example 3.20 Let $V=\operatorname{span}_{\mathbf{k}}\left\{x^{2}, x y, y^{2}, x^{\prime} y-x y^{\prime}\right\} \subset \mathbf{k}\{x, y\}$, and let us consider the following representation $\rho: \mathrm{PSL}_{2} \rightarrow \mathrm{GL}(V)$ (cf. [34, Example 3.7]):

$$
\left(\begin{array}{ll}
a & b \\
c & d
\end{array}\right) \bmod \left\{\left(\begin{array}{ll}
1 & 0 \\
0 & 1
\end{array}\right),\left(\begin{array}{cc}
-1 & 0 \\
0 & -1
\end{array}\right)\right\} \mapsto\left(\begin{array}{cccc}
a^{2} & a b & b^{2} & a^{\prime} b-a b^{\prime} \\
2 a c & a d+b c & 2 b d & 2\left(b c^{\prime}-a d^{\prime}\right) \\
c^{2} & c d & d^{2} & c^{\prime} d-c d^{\prime} \\
0 & 0 & 0 & 1
\end{array}\right)
$$

Note that $\overline{\rho\left(\mathrm{PSL}_{2}\right)}=\mathbf{G}_{a}^{3} \rtimes \mathrm{PSL}_{2}$, and we have: $R_{u}\left(\mathrm{PSL}_{2}\right)=\{e\}$ whereas $R_{u}\left(\mathbf{G}_{a}^{3} \rtimes\right.$ $\left.\mathrm{PSL}_{2}\right)=\mathbf{G}_{a}^{3}$. By [49, Theorem 1.1 and Lemma 2.2], we can construct a $\partial$-module $\mathscr{U}$ such that $\mathrm{Gal}^{\delta}(\mathscr{U})=\mathrm{PSL}_{2}$, and $\rho$ is the representation of $\mathrm{Gal}^{\delta}(\mathscr{U})$ on $\omega(\mathscr{U})$ (so that $\left.\operatorname{Gal}(\mathscr{U})=\mathbf{G}_{a}^{3} \rtimes \mathrm{PSL}_{2}\right)$. We can also construct a $\partial$-module $\mathscr{L}$ such that $\mathscr{U}$ is an extension of 1 by $\mathscr{L}$ in the given representation.

For a subset $B$ of a $\mathbf{k}$-vector space $V$, we denote $\mathbf{k} B$ the smallest $\mathbf{k}$-subspace of $V$ that contains $B$. Note that $\mathbf{k} B$ consists of all finite linear combinations of elements of $B$ with coefficients in $\mathbf{k}$.

Proposition 3.21 Let $G$ be a reductive linear differential algebraic group and $V$ a purely non-constant completely reducible $G$-module. Then every $G$-invariant $\delta$ subgroup $A \subset V$ is a submodule.

Proof We need only to show that $A$ is k-invariant. Let us assume that $G$ is connected. The general case will follow by Propositions 2.23 and 2.54, which imply that $V$ is completely reducible and purely non-constant as a $G^{\circ}$-module. 
Let us prove that $A$ is $\mathbf{k}$-invariant by induction on $\operatorname{dim} V$. Let $B$ be minimal among the non-zero $G$-invariant $\delta$-subgroups of $V$ that are contained in $A$, which exists by the Ritt-Noetherianity of the Kolchin topology. In what follows, we shall prove that $\mathbf{k} B=B$. Assuming this, by the semisimplicity of $V$, let $W \subset V$ be a $G$-invariant ksubspace such that $V=B \oplus W$. Then $A=B \oplus(W \cap A)$, and $\mathbf{k}(W \cap A)=W \cap A$ by the inductive hypothesis. Therefore, $\mathbf{k} A=A$.

Let us show that there exists $x \in \mathbf{k} \backslash C$ such that $x B=B$. Since $V$ is purely nonconstant, $V^{\prime}=\mathbf{k} B$ is purely non-constant, and so it contains a simple non-constant submodule $U$. By Corollary 2.29, there exists a $\delta$-torus $T \subset G$ such that $U$ semisimple and non-constant as a $T$-module. By the construction of $T$ (see the proof of Corollary 2.29) and Proposition 2.27, every simple $G$-module is semisimple as a $T$-module. Therefore, $V$ and $V^{\prime}$ are semisimple as $T$-modules. Hence, $\bar{T}$ is an algebraic torus, and there is a direct sum of weight spaces

$$
V^{\prime}=\bigoplus_{\chi} V_{\chi}^{\prime}
$$

over all algebraic characters $\chi: \bar{T} \rightarrow \mathbf{k}^{\times}$. By definition,

$$
V_{\chi}^{\prime}=\left\{v \in V^{\prime} \mid t(v)=\chi(t) v \text { for all } t \in \bar{T}\right\} .
$$

Note that $V_{\chi}^{\prime}$, viewed as $C$-linear spaces, are weight spaces with respect to $\bar{T}(C)=T_{C}$. Since any character $\chi$ (being defined by monomials) is uniquely determined by its restriction to $\bar{T}(C)$, the direct sum (3.13) is also the weight space decomposition of the $C$-space $V^{\prime}$ with respect to the action of $T_{C}$. Since $T_{C} \subset T \subset G$ and the $\delta$-subgroup $B \subset V^{\prime}$ is $G$-invariant, $B$ is also $T_{C}$-invariant. Moreover, $B$ is a $C$-vector space [8, Proposition 11]. Therefore, we have the weight decomposition of the $C$-space with respect to the action of $T_{C}$ :

$$
B=\bigoplus_{\chi} B_{\chi}, \quad \text { where } \quad B_{\chi}=\left(B \cap V_{\chi}^{\prime}\right) .
$$

Since $V^{\prime}=\mathbf{k} B, V_{\chi}^{\prime}=\mathbf{k} B_{\chi}$. In particular, $B_{\chi}$ is non-zero if $V_{\chi}^{\prime}$ is. By the definition of $T$, there is a character $\chi$ of $\bar{T}$ such that $\chi(T) \not \subset C$ and $V_{\chi}^{\prime} \neq\{0\}$. Therefore, there exist $b \in B_{\chi}, b \neq 0$, and $t \in T$ such that $t$ acts on $b$ by multiplication by a non-constant element $x$. We fix such an $x$. Due to the $G$-invariance of $x B$, we obtain that $B \cap x B$ is a $G$-invariant non-trivial $\delta$-subgroup of $B$. Since $B$ is minimal, $x B=B$. hand,

On the one hand, the set $S=\{a \in \mathbf{k} \mid a B \subset B\}$ is a $C$-subalgebra of $\mathbf{k}$. On the other

$$
S=\bigcap_{b \in B} \varphi_{b}^{-1}(B), \quad \varphi_{b}: \mathbf{k} \rightarrow V, \quad t \mapsto t b,
$$

is a $\delta$-subgroup of $\mathbf{k}$. Therefore, by [29, Theorem II.6.3, page 97], $S=C$ or k. Since $x \in S, S=\mathbf{k}$.

Proof (Proof of Theorem 3.19) By Theorem 3.13, $R_{u}\left(\mathrm{Gal}^{\delta}(\mathscr{U})\right.$ ) is a $\delta$-closed subgroup $W$ of $\omega(\mathscr{L})$ which is stable under the action of $\mathrm{Gal}^{\delta}(\mathscr{L})$. Proposition 3.21 shows that $W$ is a $\mathbf{k}$-vector space and thereby a $\mathrm{Gal}^{\delta}(\mathscr{L})$-module. By $\delta$-Tannakian equivalence for 
the category $\{\mathscr{L}\}^{\otimes, \delta}$, we obtain that $W$ is of the form $\omega(\mathscr{W})$ for some $\partial$-submodule $\mathscr{W} \subset \mathscr{L} \subset \mathscr{U}$. Thus, it remains to prove that $\mathscr{W}$ is the smallest $\partial$-submodule $\widetilde{L}_{0}$ of $\mathscr{L}$ such that the parameterized differential Galois group of $\mathscr{U} / \widetilde{\mathscr{L}}_{0}$ is reductive.

Let us show that the set $\mathbf{V}$ of subobjects $\mathscr{W}$ of $\mathscr{L}$ such that $R_{u}\left(\mathrm{Gal}^{\delta}(\mathscr{U} / \mathscr{W})\right)=\{1\}$ admits a smallest subobject with respect to the inclusion. It is enough to prove that, if $\mathscr{V}_{1}$ and $\mathscr{V}_{2}$ belong to $\mathbf{V}$, their intersection $\mathscr{W}$ lies in $\mathbf{V}$. Denote by $G, G_{1}$, and $G_{2}$ the parameterized differential Galois groups of $\mathscr{U} / \mathscr{W}, \mathscr{U} / \mathscr{V}_{1}$, and $\mathscr{U} / \mathscr{V}_{2}$, respectively. The quotient maps $\mathscr{U} / \mathscr{W} \rightarrow \mathscr{U} / \mathscr{V}_{i}$ give rise to homomorphisms $\varphi_{i}: G \rightarrow G_{i}, i=1,2$. Since $G_{i}$ are reductive, $R_{u}(G) \subset \operatorname{ker} \varphi_{i}$. Therefore, it suffices to show that $\operatorname{ker} \varphi_{1} \cap \operatorname{ker} \varphi_{2}=\{1\}$. For each $g \in G$, the condition $g \in \operatorname{ker} \varphi_{i}$ means that $g(u)-u \in \omega\left(\mathscr{V}_{i}\right)$ for all $u \in \omega(\mathscr{U})$. Therefore, every element of $\operatorname{ker} \varphi_{1} \cap \operatorname{ker} \varphi_{2}$ acts trivially on $\omega(\mathscr{U}) / \omega(\mathscr{W})$.

As in the notation of Lemma 3.6, let $s$ be a k-linear section of the last arrow of the following exact sequence

$$
0 \rightarrow \omega(\mathscr{L}) \rightarrow \omega(\mathscr{U}) \rightarrow \mathbf{k} \rightarrow 0
$$

and let $\zeta_{\mathscr{U}}$ be its associated cocycle. By Lemma 3.6 and Proposition 3.21, the cocycle $\zeta_{\mathscr{U}}$ identifies $R_{u}\left(\mathrm{Gal}^{\delta}(\mathscr{U})\right)$ with a k-vector subgroup $W=\omega(\mathscr{W})$ of $\omega(\mathscr{L})$ for some $\partial$-submodule $\mathscr{W} \subset \mathscr{U}$. To conclude the proof, we have to show that $W=\omega\left(\widetilde{\mathscr{L}}_{0}\right)$.

It follows from the definition of $\zeta$ that the diagram

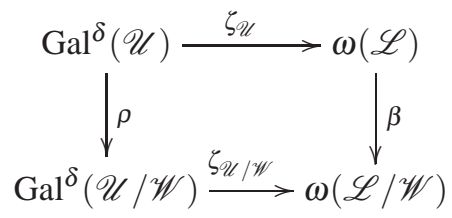

where the vertical arrows are induced by the quotient maps, is commutative. By the definition of $\mathscr{W}$ and exactness of $\omega$, the composition $\beta \zeta_{\mathscr{U}}$ vanishes on $R_{u}\left(\operatorname{Gal}^{\delta}(\mathscr{U})\right)$. Since $\omega(\mathscr{U} / \mathscr{W})$ is a faithful $\mathrm{Gal}^{\delta}(\mathscr{U} / \mathscr{W})$-module and $\omega(\mathscr{L} / \mathscr{W})$ has no non-zero trivial $\mathrm{Gal}^{\delta}(\mathscr{L} / \mathscr{W})$-submodule by assumption, and therefore no non-zero trivial $\mathrm{Gal}^{\delta}(\mathscr{U} / \mathscr{W})$-submodules by assumption, Propositions 3.22 and 3.23 below show that

$$
R_{u}\left(\mathrm{Gal}^{\delta}(\mathscr{U} / \mathscr{W})\right)=\rho\left(R_{u}\left(\mathrm{Gal}^{\delta}(\mathscr{U})\right)\right)
$$

Since $\zeta$ is one-to-one on the unipotent radical, we conclude that the linear differential algebraic group $\operatorname{Gal}^{\delta}(\mathscr{U} / \mathscr{W})$ is reductive. Therefore, $\mathscr{W} \supset \widetilde{\mathscr{L}}_{0}$. If we replace $\mathscr{W}$ with a $\partial$-submodule $\mathscr{V} \subset \mathscr{U}$ in the above diagram such that $\mathrm{Gal}^{\delta}(\mathscr{U} / \mathscr{V})$ is reductive, we obtain that

$$
\omega(\mathscr{V}) \supset \zeta_{\mathscr{U}}\left(R_{u}\left(\operatorname{Gal}^{\delta}(\mathscr{U})\right)\right)=W
$$

Thus, $\omega\left(\widetilde{\mathscr{L}_{0}}\right) \supset W$.

Recall that unipotent linear differential algebraic groups are connected. (Otherwise they would have unipotent finite quotients, which is impossible.) Therefore, for every linear differential algebraic group $G$, we have $R_{u}(G)=R_{u}\left(G^{\circ}\right)=R_{u}(G)^{\circ}$. 
Proposition 3.22 Let $\rho: G \rightarrow H$ be a surjective homomorphism of linear differential algebraic groups. Assume that, for every proper subgroup $N \subset R_{u}(H)$ that is normal in $H$, the group $R_{u}(H / N)$ is not central in $(H / N)^{\circ}=H^{\circ} / N$. Then $\rho\left(R_{u}(G)\right)=R_{u}(H)$.

Proof Let $N=\rho\left(R_{u}(G)\right) \subset R_{u}(H)$. By the surjectivity of $\rho$, the group $N$ is normal in $H$. Consider the epimorphism of quotients

$$
v: G / R_{u}(G) \rightarrow H / N
$$

induced by $\rho$. The linear differential algebraic group $v^{-1}\left(R_{u}(H / N)\right)^{\circ}$ is normal in the reductive linear differential algebraic group $\left(G / R_{u}(G)\right)^{\circ}$. Therefore, it is reductive itself. By Theorem 2.25, $v^{-1}\left(R_{u}(H / N)\right)^{\circ}$ is an almost direct product of a $\delta$-closed subgroup $Z$ of a central torus $T \subset\left(G / R_{u}(G)\right)^{\circ}$ and of quasi-simple linear differential algebraic groups $H_{i}$. Since the subgroups $H_{i}$ coincide with their commutator groups, they cannot have unipotent images unless $v\left(H_{i}\right)=\{e\}$. We conclude that $v(Z)=R_{u}(H / N)$. Since $Z$ is central in $\left(G / R_{u}(G)\right)^{\circ}$ and $v$ is surjective, the group $v(Z)$ is central in $(H / N)^{\circ}$. It follows from the assumption that $N=R_{u}(H)$.

Proposition 3.23 The assumption on $H$ in Proposition 3.22 is satisfied if there exists a short exact sequence

$$
0 \rightarrow V \rightarrow U \rightarrow \mathbf{1} \rightarrow 0
$$

of $\mathrm{H}^{\circ}$-modules, where $U$ is a faithful $\mathrm{H}^{\circ}$-module and $V$ is a $\mathrm{H}^{\circ}$-semisimple module with no non-zero trivial $H^{\circ}$-submodule.

Remark 3.24 Note that if the $H^{\circ}$-module $V$ has no trivial $H^{\circ}$-submodules, then $V$ has no no zero $C$-vector space fixed by the action of $H^{\circ}$. Indeed, let $f$ be a nonzero element of a $C$-vector space fixed by $H^{\circ}$, then the $\mathbf{k}$-vector space spanned by $f$ is fixed by $H^{\circ}$.

Proof It suffices to prove the statement for connected $H$. Let $N \subset R_{u}(H)$ be a $\delta$ subgroup that is normal in $H$ and such that $R_{u}(H / N)$ is central in $H / N$. Since we have a commutative diagram

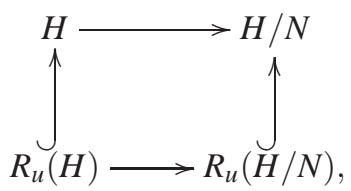

the latter implies that, for all $g \in R_{u}(H)$, one has $h g h^{-1} \in g N$. Let $u \in U$ be an element whose image in $\mathbf{1}$ is non-zero. Moreover, $R_{u}(H)$ acts trivially on $V$ because $V$ is $H$ semi-simple. Thus, the map

$$
\zeta: R_{u}(H) \rightarrow V, \quad g \mapsto g u-u
$$

is an $H$-equivariant one-to-one homomorphism of linear differential algebraic groups (see proofs of Lemmas 3.6 and 3.8), that is, for all $h \in H$ and $g \in R_{u}(H)$, we have

$$
h g u-h u=h g h^{-1} u-u .
$$


The $\delta$-subgroups $\zeta\left(R_{u}(H)\right)$ and $\zeta(N)$ of $V$ are thus stable under the action of $H$. Note that $\zeta\left(R_{u}(H)\right)$ and $\zeta(N)$ are $C$-vector spaces since, as $\delta$-subgroup of $V$, they are zero sets linear homogeneous differential equations over $\mathbf{k}$.

Let $n \in N$ be such that $h g h^{-1}=g n$ and $n^{\prime} \in N$ be such that $g n g^{-1}=n^{\prime}$. Then

$$
\begin{aligned}
h(g u-u) & =h g u-h u=g n u-u=n^{\prime} g u-u+n^{\prime} u-n^{\prime} u \\
& =n^{\prime}(g u-u)+n^{\prime} u-u=g u-u+n^{\prime} u-u,
\end{aligned}
$$

since $g u-u \in V$ and $R_{u}(H)$ acts trivially on $V$. Therefore, $H$ acts trivially on $\zeta\left(R_{u}(H)\right) / \zeta(N)$. Since $\zeta\left(R_{u}(H)\right)$ is $H$-semisimple as $H$-module over $C$, the $H$-module

$$
\zeta\left(R_{u}(H)\right) / \zeta(N) \subset \zeta\left(R_{u}(H)\right) \subset V
$$

is a $C$-vector space fixed by the action of $H$. This contradicts the assumption on $V$. It follows that $R_{u}(H)=N$.

\subsubsection{A general algorithm}

Will will explain a general algorithm to compute the unipotent radical of a $\partial$-module extension $\mathscr{U}$ of $\mathbf{1}$ by a completely reducible $\partial$-module $\mathscr{L}$. We recall that $\mathscr{L}$ can be decomposed as the direct sum of a constant $\partial$-module $\mathscr{L}_{c}$ and a purely non-constant $\partial$-module $\mathscr{L}_{n c}$. Considering the pushouts of the extension $\mathscr{U}$ with respect to the decomposition of $\mathscr{L}$, we find the following two exact sequences of $\partial$-modules:

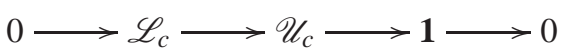

and

$$
0 \longrightarrow \mathscr{L}_{n c} \longrightarrow \mathscr{U}_{n c} \longrightarrow \mathbf{1} \longrightarrow 0
$$

We assume that $K=\mathbf{k}(x)$ so that we can use the algorithm contained in [35] to compute $R_{u}\left(\mathrm{Gal}^{\delta}\left(\mathscr{U}_{c}\right)\right)$ and the algorithm of Section 3.3.2 to compute $R_{u}\left(\mathrm{Gal}^{\delta}\left(\mathscr{U}_{n c}\right)\right)$. The quotient map $\mathscr{U} \rightarrow \mathscr{U} / \mathscr{U}_{c}=\mathscr{U}_{n c}$ induces an epimorphism $\alpha: \operatorname{Gal}^{\delta}(\mathscr{U}) \rightarrow \operatorname{Gal}^{\delta}\left(\mathscr{U}_{n c}\right)$. Similarly, we find an epimorphism $\beta: \operatorname{Gal}^{\delta}(\mathscr{U}) \rightarrow \operatorname{Gal}^{\delta}\left(\mathscr{U}_{c}\right)$. The following theorem allows us to compare $R_{u}\left(\mathrm{Gal}^{\delta}(\mathscr{U})\right)$ with the groups computed above.

Theorem 3.25 Let $K=\mathbf{k}(x), \mathscr{L}, \mathscr{U}, \mathscr{U}_{c}, \mathscr{U}_{n c}$ be as above. Assume that $\mathscr{L}$ has no nonzero trivial $\partial$-submodule. Then the map

$$
\alpha \times \beta: R_{u}\left(\mathrm{Gal}^{\delta}(\mathscr{U})\right) \rightarrow R_{u}\left(\mathrm{Gal}^{\delta}\left(\mathscr{U}_{n c}\right)\right) \times R_{u}\left(\mathrm{Gal}^{\delta}\left(\mathscr{U}_{c}\right)\right)
$$

is an isomorphism of linear differential algebraic groups.

Proof We will use the notion of differential type $\tau(G)$ of a linear differential algebraic group $G$ (see [12, Section 2.1] and [35, Definition 2.2]). Recall that, in the ordinary case, $\tau$ can only take the values $-1,0$, or 1 . We will also use the following result:

Lemma 3.26 ([12, Equation (1), page 195]) Let $G$ be a linear differential algebraic group and $H$ be a normal differential algebraic subgroup of $G$. Then $\tau(G)=$ $\max \{\tau(H), \tau(G / H)\}$. 
Let us consider the commutative diagram:

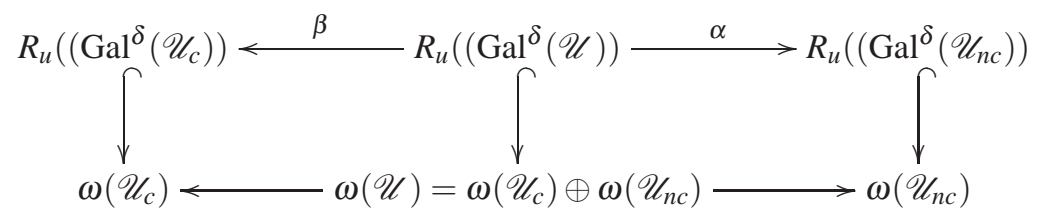

Here, the vertical arrows correspond to embedding (that is, a one-to-one homomorphism) via the associated cocycles (see (3.14)). The horizontal arrows of the lower row correspond to natural projections. Note that $R_{u}\left(\left(\mathrm{Gal}^{\delta}\left(\mathscr{U}_{c}\right)\right), R_{u}\left(\left(\mathrm{Gal}^{\delta}(\mathscr{U})\right)\right.\right.$, and $R_{u}\left(\left(\mathrm{Gal}^{\delta}\left(\mathscr{U}_{n c}\right)\right)\right.$ are all abelian groups (see Theorem 3.3). It follows from (3.15) that $\alpha \times \beta$ is an embedding. Then, by [12, Corollary 2.4] and Lemma 3.26,

$$
\begin{aligned}
\tau\left(R_{u}\left(\mathrm{Gal}^{\delta}(\mathscr{U})\right)\right. & \leq \tau\left(R_{u}\left(\mathrm{Gal}^{\delta}\left(\mathscr{U}_{c}\right)\right) \times R_{u}\left(\mathrm{Gal}^{\delta}\left(\mathscr{U}_{n c}\right)\right)\right) \\
& =\max \left\{\tau\left(R_{u}\left(\mathrm{Gal}^{\delta}\left(\mathscr{U}_{c}\right)\right)\right), \tau\left(R_{u}\left(\mathrm{Gal}^{\delta}\left(\mathscr{U}_{n c}\right)\right)\right)\right\} .
\end{aligned}
$$

Since $\alpha$ and $\beta$ are surjective, we find that

$$
\tau\left(R_{u}\left(\mathrm{Gal}^{\delta}(\mathscr{U})\right)=\max \left\{\tau\left(R_{u}\left(\mathrm{Gal}^{\delta}\left(\mathscr{U}_{c}\right)\right)\right), \tau\left(R_{u}\left(\mathrm{Gal}^{\delta}\left(\mathscr{U}_{n c}\right)\right)\right)\right\} .\right.
$$

If $R_{u}\left(\mathrm{Gal}^{\delta}\left(\mathscr{U}_{n c}\right)\right) \neq\{e\}$, it is isomorphic to a non-trivial vector group over $\mathbf{k}$ and its differential type is 1 (see [12, Example 2.9]). Moreover, since the unipotent radicals considered above are $\delta$-closed subgroups of vector groups, they are either algebraic groups and their differential type is 1 , or finite-dimensional $C$-vector spaces of differential type 0 . If $R_{u}\left(\mathrm{Gal}^{\delta}\left(\mathscr{U}_{n c}\right)=\{e\}\right.$, we have nothing to prove. Thus, we assume that $R_{u}\left(\mathrm{Gal}^{\delta}\left(\mathscr{U}_{n c}\right) \neq\{e\}\right.$ and that its differential type is 1 . By the discussion above, we can also assume that

$$
\tau\left(R_{u}\left(\mathrm{Gal}^{\delta}(\mathscr{U})\right)\right)=1 .
$$

Since $\mathscr{L}$ has no non-zero trivial $\partial$-submodule, the same holds for $\mathscr{L}_{c}$ and $\mathscr{L}_{n c}$. By Propositions 3.22 and 3.23, $\alpha$ and $\beta$ are surjective. Let $R_{0} \subset R_{u}\left(\mathrm{Gal}^{\delta}(\mathscr{U})\right)$ stand for the strong identity component of $R_{u}\left(\mathrm{Gal}^{\delta}(\mathscr{U})\right)$ ([12, Definition 2.6]). Since $R_{u}\left(\mathrm{Gal}^{\delta}\left(\mathscr{U}_{n c}\right)\right)$ is algebraic by Theorem 3.19, it is strongly connected by [12, Lemma 2.8 and Example 2.9]. We have

$$
\alpha\left(R_{0}\right)=R_{u}\left(\mathrm{Gal}^{\delta}\left(\mathscr{U}_{n c}\right)\right)
$$

(Indeed, otherwise $\alpha\left(R_{0}\right) \subsetneq R_{u}\left(\mathrm{Gal}^{\delta}\left(\mathscr{U}_{n c}\right)\right)$. By definition of the strong identity component, we find that

$$
\tau\left(R_{u}\left(\mathrm{Gal}^{\delta}(\mathscr{U})\right) / R_{0}\right)<1 .
$$

However,

$$
\tau\left(R_{u}\left(\mathrm{Gal}^{\delta}\left(\mathscr{U}_{n c}\right)\right) / \alpha\left(R_{0}\right)\right)=1
$$

because $R_{u}\left(\mathrm{Gal}^{\delta}\left(\mathscr{U}_{n c}\right)\right)$ is strongly connected. Therefore, we have a surjective map

$$
R_{u}\left(\mathrm{Gal}^{\delta}(\mathscr{U})\right) / R_{0} \rightarrow R_{u}\left(G_{n c}\right) / \alpha\left(R_{0}\right)
$$


from a linear differential algebraic group of differential type smaller than 1 onto a linear differential algebraic group of differential type 1 , which is impossible. Therefore, the group product map

$$
R_{0} \times \operatorname{ker} \alpha \rightarrow R_{u}\left(\operatorname{Gal}^{\delta}(\mathscr{U})\right), \quad\left(r_{0}, x\right) \mapsto r_{0} x
$$

is onto. To finish the proof, it suffices to show that

$$
\beta(\operatorname{ker} \alpha)=R_{u}\left(\operatorname{Gal}^{\delta}\left(\mathscr{U}_{c}\right)\right) .
$$

If $\beta\left(R_{0}\right) \neq\{e\}$, it is strongly connected and

$$
\tau\left(\beta\left(R_{0}\right)\right)=\tau\left(R_{0}\right)=1
$$

Since $\tau\left(R_{u}\left(\operatorname{Gal}^{\delta}\left(\mathscr{U}_{n c}\right)\right)\right)=0$ (see [35, Theorem 2.13]), we have $\beta\left(R_{0}\right)=\{e\}$ (by Lemma 3.26). Thus,

$$
\beta(\operatorname{ker} \alpha)=R_{u}\left(\mathrm{Gal}^{\delta}\left(\mathscr{U}_{n c}\right)\right)
$$

\section{Criteria of hypertranscendance}

We start with a new result in the representation theory of quasi-simple and reductive linear differential algebraic groups, which we further use for a hypertranscendence criterion.

\subsection{Extensions of the trivial representation}

Let $(\mathbf{k}, \delta)$ be a $\delta$-closed field such that chark $=0$ and let $C$ be its field of $\delta$-constants. Let $G \subset \mathrm{GL}_{n}(\mathbf{k})$ be a connected linear differential algebraic group over $\mathbf{k}$. We recall the definition of the Lie algebra of $G$, following [8, Chapter 3].

Definition 4.1 A k-linear derivation $D$ of the field of fractions $\mathbf{k}\langle G\rangle$ of the $\delta$-coordinate ring $\mathbf{k}\{G\}$ of $G$ is called a differential derivation if $D \circ \delta=\delta \circ D$.

In particular, every differential derivation is determined by its values on the matrix entries that differentially generate $\mathbf{k}\{G\}$ and, therefore, can be represented by an $n \times n$ matrix. The group $G$ acts by right translations on the set of differential derivations of $\mathbf{k}\langle G\rangle$.

Definition 4.2 The set Lie $G$ of invariant differential derivations, denoted also by $\mathfrak{g}$, is called the Lie algebra of $G$.

This is a $C$-Lie subalgebra of the Lie algebra $\mathfrak{g l}_{n}(\mathbf{k})=\operatorname{LieGL}_{n}(\mathbf{k})$ of all $n \times n$ matrices. Moreover, $\mathfrak{g}$ is also a $\delta$-subgroup of the additive group of $\mathfrak{g l}_{n}(\mathbf{k})$. Every $\delta$ homomorphism of linear differential algebraic groups gives rise (by taking the differential) to a $C$-homomorphism of their Lie algebras. We refer to [8, Chapter 3] for the details. 
Definition 4.3 A $\mathfrak{g}$-module (respectively, $C$ - $\mathfrak{g}$-module) is a finite-dimensional $\mathbf{k}$-vector space (respectivelty, $C$-vector space, possibly infinite-dimensional) $V$ together with a $C$-Lie algebra homomorphism $v: \mathfrak{g} \rightarrow \mathfrak{g l}(V)$, where $\mathfrak{g l}(V)$ denotes the Lie algebra of k-linear endomorphisms of $V$.

Every $G$-module $V$ is also a $\mathfrak{g}$-module, where $v=d \rho: \mathfrak{g} \rightarrow \mathfrak{g l}(V)$ is the differential (see [8, pages 928-929]) of the homomorphism $\rho: G \rightarrow \mathrm{GL}(V)$. (Formally, to agree with the above definitions, we assume that a basis of $V$ is chosen, hence we can identify $\mathrm{GL}(V)$ and $\mathfrak{g l}(V)$ with $\mathrm{GL}_{n}(\mathbf{k})$ and $\mathfrak{g l}_{n}(\mathbf{k})$, respectively.) The definitions of simple, semisimple, and other types of $\mathfrak{g}$-modules that we use here are analogues to those for $G$-modules.

It follows from [8, Proposition 20] that, if $G \subset \mathrm{GL}_{n}(\mathbf{k})$ is given by polynomial equations, then Lie $G$ coincides with the Lie algebra of the group $G$ considered as an algebraic group. Moreover, for an arbitrary linear differential algebraic group $G \subset \mathrm{GL}_{n}(\mathbf{k})$, the Lie algebra Lie $\bar{G}$ of its Zariski closure $\bar{G}$ coincides with the k-span of Lie $G$ in $\mathfrak{g l}_{n}(\mathbf{k})$. Recall that, in the case of $G=\bar{G}, \operatorname{Lie} G$ is a $G$-module, which is called adjoint, where the action of $G$ is induced from its action on $\mathfrak{g l}_{n}(\mathbf{k})$ by conjugation. The differential of the corresponding homomorphism $\mathrm{Ad}: G \rightarrow \mathrm{GL}(\mathfrak{g})$ gives the k-Lie algebra map ad $: \mathfrak{g} \rightarrow \mathfrak{g l}(\mathfrak{g})$ defining the structure of the $\mathfrak{g}$-module on $\mathfrak{g}$, also called adjoint. One has $(\operatorname{ad} x)(y)=[x, y]$ for all $x, y \in \mathfrak{g}$.

For any group, Lie algebra, or ring $R$, we denote the set of $R$-module homomorphisms by $\operatorname{Hom}_{R}(V, W)$.

For a $C$-Lie algebra $\mathfrak{g}$, let $\mathfrak{g}_{\mathbf{k}}=\mathbf{k} \otimes_{C} \mathfrak{g}$ denote the $\mathbf{k}$-Lie algebra with the bracket determined by

$$
[x \otimes \xi, y \otimes \eta]=x y \otimes[\xi, \eta] \quad \forall x, y \in \mathbf{k}, \quad \xi, \eta \in \mathfrak{g} .
$$

We have the inclusion

$$
\mathfrak{g} \simeq C \otimes \mathfrak{g} \subset \mathbf{k} \otimes \mathfrak{g}=\mathfrak{g}_{\mathbf{k}} .
$$

If $\mathfrak{g} \subset \mathfrak{h}$ are Lie algebras, then we also consider $\mathfrak{h}$ as a $\mathfrak{g}$-module under the adjoint action.

Lemma 4.4 Let $H \subset \mathrm{GL}_{n}(C)$ be a reductive algebraic group and $\mathfrak{h}=\operatorname{Lie} H \subset \mathfrak{g l}_{n}(C)$. Let $\mathfrak{g} \subset \mathfrak{h}_{k}$ be a $C$-Lie subalgebra containing $\mathfrak{h}$ and

$$
0 \rightarrow V \rightarrow W \rightarrow \mathbf{1} \rightarrow 0
$$

an exact sequence of $\mathfrak{g}$-modules (over $\mathbf{k})$. If

(1) sequence (4.1) splits as a sequence of $\mathfrak{h}$-modules and

(2) $\operatorname{Hom}_{\mathfrak{h}_{\mathbf{k}}}\left(\mathfrak{h}_{\mathbf{k}}, V\right)=0$ (in other words, $V$ does not contain quotients of the adjoint representation of $\left.\mathfrak{h}_{\mathbf{k}}\right)$,

then sequence (4.1) splits.

Proof If one chooses a basis $\left\{e_{1}, \ldots, e_{n-1}, e_{n}\right\}$ of $W$ such that $V=\operatorname{span}\left\{e_{1}, \ldots, e_{n-1}\right\}$, then the matrix $\rho(\xi) \in \mathfrak{g l}(W)$ corresponding to $\xi \in \mathfrak{g}$ can be written in the form

$$
\left(\begin{array}{cc}
\alpha(\xi) & \varphi(\xi) \\
0 & 0
\end{array}\right)
$$


where $\alpha: \mathfrak{g} \rightarrow \mathfrak{g l}(V)$ determines the $\mathfrak{g}$-module structure on $V$ and $\varphi: \mathfrak{g} \rightarrow V$ is a $C$ linear map. The fact that $\rho$ defines a homomorphism of Lie algebras is the following condition on $\varphi$ :

$$
\varphi([\xi, \eta])=\alpha(\xi) \varphi(\eta)-\alpha(\eta) \varphi(\xi) \quad \forall \xi, \eta \in \mathfrak{g} .
$$

Choosing another vector for $e_{n}$, one obtains another $C$-linear map $\varphi^{\prime}: \mathfrak{g} \rightarrow V$, which is called equivalent to $\varphi$. Sequence (4.1) splits if and only if $\varphi$ is equivalent to 0 .

Let us choose $e_{n}$ in such a way that

$$
\varphi(\xi)=0 \quad \forall \xi \in \mathfrak{h},
$$

which is possible due to assumption (1). It follows from (4.2) and (4.3) that

$$
\varphi([\xi, \eta])=\alpha(\xi) \varphi(\eta) \quad \forall \xi \in \mathfrak{h}, \quad \eta \in \mathfrak{g} .
$$

Since $H$ is reductive, by [52, page 97, Theorem] and [50, Chapter 2], there exist simple $\mathfrak{h}$-submodules $\mathfrak{h}_{1}, \ldots, \mathfrak{h}_{m}$ in $\mathfrak{h}$ such that $\mathfrak{h}=\bigoplus_{i=1}^{m} \mathfrak{h}_{i}$. Let $B \subset \mathbf{k}$ be a $C$-basis of $\mathbf{k}$ as a $C$-vector space. For each $a \in \mathbf{k}$ and $i, 1 \leq i \leq m, a \otimes \mathfrak{h}_{i}$ is a simple $C$-h $\mathfrak{h}$-submodule of $\mathfrak{h}_{\mathbf{k}}$ and

$$
\mathfrak{h}_{\mathbf{k}}=\bigoplus_{\substack{1 \leq i \leq m \\ b \in B}} b \otimes \mathfrak{h}_{i} .
$$

For every $C$-h $\mathfrak{h}$-submodule $I \subset \mathfrak{h}_{\mathbf{k}}$, let $I^{\prime}$ be a maximal sum of the simple components in decomposition (4.5) with $I^{\prime} \cap I=\{0\}$. Such an $\mathfrak{h}$-submodule $I^{\prime}$ exists by Zorn's lemma. We will show that

$$
\mathfrak{h}_{\mathbf{k}}=I \oplus I^{\prime}
$$

Let $S=b \otimes \mathfrak{h}_{i}$ for some $b \in B$ and $1 \leq i \leq m$. If $S \cap\left(I \oplus I^{\prime}\right)=\{0\}$, then $I \cap\left(S \oplus I^{\prime}\right)=\{0\}$. Indeed, if $v \in I$ and $v=v_{1}+v_{2}$, where $v_{1} \in S$ and $v_{2} \in I^{\prime}$, then $v_{2}=v-v_{1} \in S \cap\left(I \oplus I^{\prime}\right)$, and so $v=v_{1} \in I \cap S=\{0\}$. By the maximality of $I^{\prime}, S \subset I^{\prime}$, which contradicts $S \cap(I \oplus$ $\left.I^{\prime}\right)=\{0\}$. Therefore,

$$
S \cap\left(I \oplus I^{\prime}\right) \neq\{0\} .
$$

Since $S$ is a simple $\mathfrak{h}$-module, (4.7) implies that $S \subset I \oplus I^{\prime}$. Thus, (4.6) holds and therefore $\mathfrak{h}_{\mathbf{k}}$ is a semisimple $\mathfrak{h}$-module. (cf. [7, §4.1]).

The $C$-h-module $\mathfrak{g}$ is semisimple. Indeed, every $\mathfrak{h}$-invariant subspace $J \subset \mathfrak{g}$ has a complementary invariant subspace $J^{\prime}$ in $\mathfrak{h}_{k}$, since $\mathfrak{h}_{k}$ is semisimple. Therefore,

$$
\mathfrak{g}=J \oplus\left(J^{\prime} \cap \mathfrak{g}\right) .
$$

Thus, to prove that $\varphi$ is the zero map, it suffices to show that $\varphi(J)=\{0\}$ for every simple $C$-h-h-submodule $J \subset \mathfrak{g}$. Since such $J$ is isomorphic to $\mathfrak{h}_{i}$ for some $i, 1 \leq i \leq m$, we have the $\mathfrak{h}$-equivariant $C$-linear map

$$
\mu: \mathfrak{h} \stackrel{\pi}{\rightarrow} \mathfrak{h}_{i} \simeq J \subset \mathfrak{g} \stackrel{\varphi}{\rightarrow} V,
$$

where $\pi$ is the projection with respect to an $\mathfrak{h}$-invariant decomposition $\mathfrak{h}=\mathfrak{h}_{i} \oplus \mathfrak{h}_{i}^{\prime}$, and the $\mathfrak{h}$-equivariance of $\varphi$ is implied by (4.4). Since $\mu$ extends to the k-linear $\mathfrak{h}_{k^{-}}$ equivariant map $\mathfrak{h}_{k} \rightarrow V$, assumption (2) yields that $\mu$ is the zero map. Therefore, $\varphi(J)=\{0\}$. 
Lemma 4.5 Let $G$ be a connected linear differential algebraic group and $\mathfrak{g}$ be its Lie algebra. Any G-module $W$ is completely reducible if and only if it is completely reducible as a $\mathfrak{g}$-module.

Proof Let $G_{W}$ denote the image of $G$ in $\mathrm{GL}(W)$. The $G$-module $W$ is completely reducible if and only if it is completely reducible as a $G_{W}$-module. The latter is equivalent to $W$ being completely reducible as a $\overline{G_{W}}$-module. Since char $\mathbf{k}=0$, this is equivalent to the semisimplicity of $W$ viewed as the Lie $\overline{G_{W}}$-module (see [52, page 97, Theorem]). Since Lie $\overline{G_{W}}$ is the $\mathbf{k}$-span of $\operatorname{Lie} G_{W} \subset \mathfrak{g l}(W), W$ is completely reducible as a Lie $\overline{G_{W}}$ if and only if it is completely reducible as a Lie $G_{W}$-module. Since, by [8, Proposition 22], Lie $G_{W}$ is an image of $\mathfrak{g}$ in $\mathfrak{g l}(W), W$ is completely reducible as a $\mathfrak{g}$-module if and only if $W$ is completely reducible as a $\operatorname{Lie} G_{W}$-module.

Theorem 4.6 Let $G$ be a connected linear differential algebraic group over $\mathbf{k}$ and

$$
0 \rightarrow V \rightarrow W \rightarrow \mathbf{1} \rightarrow 0
$$

an exact sequence of G-modules, where $V$ is faithful and semisimple. Let $\bar{G}$ denote the Zariski closure of $G$ in $\mathrm{GL}(V)$. If $V$, viewed as a $\bar{G}$-module, does not contain non-zero submodules isomorphic to a quotient of the adjoint module for $\bar{G}$, that is, if

$$
\operatorname{Hom}_{\bar{G}}(\operatorname{Lie} \bar{G}, V)=0,
$$

then sequence (4.8) splits.

Proof By Lemma 4.5, it is sufficient to show that $W$ is completely reducible as a $\mathfrak{g}$ module. Since $G$ admits a faithful completely reducible representation (given by $V$ ), it is reductive. Therefore, by [33, Lemma 4.5], there is a $\delta$-isomorphism $v: \widetilde{H} \rightarrow G$, where $\widetilde{H} \subset \mathrm{GL}_{r}(\mathbf{k})$ is a $\delta$-group such that its $\delta$-subgroup $H_{C}=\widetilde{H} \cap \mathrm{GL}_{r}(C)$ is Zariski dense (the Zariski topology on $\widetilde{H}$ is induced from $\mathrm{GL}_{r}(\mathbf{k})$ ).

Let $H=v\left(H_{C}\right)$ and $\mathfrak{h}=\operatorname{Lie} H$. We will show that $\mathfrak{h}$ and $\mathfrak{g}$ satisfy the hypotheses of Lemma 4.4, which would thus yield the proof (in particular, we will identify $\mathfrak{g}$ with a subalgebra of $\mathfrak{h}_{\mathbf{k}}$ ). The differential algebraic group $H \simeq H_{C}$ is reductive. Indeed, if its unipotent radical were non-trivial, $\overline{R_{u}\left(H_{C}\right)} \cap \widetilde{H}$ would be a non-trivial normal unipotent differential algebraic subgroup of $\widetilde{H}$, which is impossible due to the reductivity of $G \simeq$ $\widetilde{H}$.

Let us show that $v$ extends to an algebraic isomorphism $\bar{v}: \overline{H_{C}} \rightarrow \bar{G}$ of the Zariski closures. By [33, Theorem 3.3], this would follow if the $G$-module $V$ is completely reducible and $\overline{H_{C}}$ is reductive. It only remains to prove the latter. Since $H_{C}$ is reductive, $C^{r}$ is a completely reducible $H_{C}$-module. Therefore, $\mathbf{k}^{r}$ is completely reducible as an $\overline{H_{C}}$-module. Thus, $\overline{H_{C}}$ is reductive.

The differential $d \bar{v}$ defines an isomorphism between $\mathbf{k}$-Lie algebras Lie $\overline{H_{C}}$ and Lie $\bar{G}$. Since Lie $H_{C} \subset \mathfrak{g l}_{r}(C)$ and any $C$-basis of $\mathfrak{g l}_{r}(C)$ is also a $\mathbf{k}$-basis of $\mathfrak{g l}_{r}(\mathbf{k})$, we obtain that any $C$-basis of Lie $H_{C}$ is $\mathbf{k}$-linearly independent. Since Lie $\overline{H_{C}}$ is the $\mathbf{k}$-span of $\mathrm{Lie} H_{C}$, we can therefore write

$$
\operatorname{Lie} \overline{H_{C}}=\mathbf{k} \otimes_{C} \operatorname{Lie} H_{C} .
$$


Applying $d \bar{v}$, this implies that

$$
\operatorname{Lie} \bar{G}=\mathbf{k} \otimes_{C} \mathfrak{h}=\mathfrak{h}_{\mathbf{k}}
$$

Therefore, we have

$$
\mathfrak{h} \subset \mathfrak{g} \subset \mathfrak{h}_{\mathbf{k}} \cdot
$$

Since every $\delta$-representation of $H_{C}$ is polynomial and $\overline{H_{C}}$ is reductive, every $\delta$ representation of $H_{C}$ is completely reducible. Therefore, $W$ is completely reducible as an $H$-module (and $\mathfrak{h}$-module), and so sequence (4.8) splits as a sequence of $\mathfrak{h}$-modules. Finally, using [52, page 97, Theorem] and Lie $\bar{G}=\mathfrak{g}_{\mathbf{k}}$, we conclude that

$$
\operatorname{Hom}_{\mathfrak{g}_{\mathbf{k}}}\left(\mathfrak{g}_{\mathbf{k}}, V\right)=\operatorname{Hom}_{\mathrm{Lie} \bar{G}}(\operatorname{Lie} \bar{G}, V)=\operatorname{Hom}_{\bar{G}}(\operatorname{Lie} \bar{G}, V)=0 .
$$

\subsection{A practical criterion of hypertranscendance}

Let $\Delta=\{\partial, \delta\}$ be a set of two derivations. Let $K$ be a $\Delta$-field such that $K^{\partial}=\mathbf{k}$ (recall that $\mathbf{k}$ is $\delta$-closed). From the results of the previous sections, we obtain the following criterion for the hypertranscendence of the solutions of $L(y)=b$, for irreducible $L \in$ $K[\partial]$.

Theorem 4.7 Let $L \in K[\partial]$ be an irreducible $\partial$-operator such that $\operatorname{Gal}(L)$ is a quasisimple linear algebraic group. Denote $n=\operatorname{ord} L$ and $m=\operatorname{dim} \operatorname{Gal}(L)$. Suppose that $m \neq n$. Let $b \in K^{*}$ and $F$ a $\Delta$-field extension of $K$ such that $F^{\partial}=\mathbf{k}$ and $F$ contains $z, a$ solution of $L(y)=b$, and $u_{1}, \ldots, u_{n}$, K-linearly independent solutions of $L(y)=0$. Then

- the functions $v_{1}, \ldots, v_{m}, z, \ldots, \partial^{n-1} z$ and all their derivatives with respect to $\delta$ are algebraically

independent over $K$, where $\left\{v_{1}, \ldots, v_{m}\right\} \subset\left\{u_{1}, \ldots, \partial^{n-1} u_{1}, \ldots, u_{n}, \ldots, \partial^{n-1} u_{n}\right\}$ is a maximal algebraically independent over $K$ subset

if and only if

- the linear differential system $\partial(B)-\delta\left(A_{L}\right)=A_{L} B-B A_{L}$, where $A_{L}$ denotes the companion matrix of $L$, has no solutions $B \in K^{n \times n}$ and

- the linear differential equation $L(y)=b$ has no solutions in $K$.

Example 4.8 If $L \in K[\partial]$ and $\operatorname{Gal}(L)=\mathrm{SL}_{n}$, where $n=\operatorname{ord} L \geq 2$, then $L$ is irreducible and $\operatorname{dim} L \neq \operatorname{dim} \operatorname{Gal}(L)=n^{2}-1$. In this situation, in Theorem 4.7, we can take

$$
\left\{v_{1}, \ldots, v_{m}\right\}=\left\{u_{1}, \ldots, \partial^{n-1} u_{1}, \ldots, u_{n-1}, \ldots, \partial^{n-1} u_{n-1}, u_{n}, \ldots, \partial^{n-2} u_{n}\right\} .
$$

Proof (Proof of Theorem 4.7) Let $\mathscr{L}$ (respectively, $\mathscr{U}$ ) be the $\partial$-module associated to $L$ (respectively, to $(\partial-\partial(b) / b) L$ ). Since the $\Delta$-field $K_{\mathscr{U}}$ generated by $u_{1}, \ldots, u_{n}, z$ in $F$ is a PPV extension for $\mathscr{U}$ over $K$, the differential transcendence degree of $K_{\mathscr{U}}$ over $K$ equals the differential dimension of $\mathrm{Gal}^{\delta}(\mathscr{U})$. Since $\mathscr{L}$ corresponds to the differential system $\partial Y=A_{L} Y$, Proposition 2.52 together with Theorem 2.25(3) imply that the first hypothesis is equivalent to $\operatorname{Gal}^{\delta}(\mathscr{L})=\operatorname{Gal}(\mathscr{L})$. 
Since $L$ is irreducible, there is no non-zero trivial $\partial$-submodule $\mathscr{N}$ of $\mathscr{L}$ such that the representation of $\mathrm{Gal}^{\delta}(\mathscr{L})$ on $\omega(\mathscr{N})$ is conjugate to constants, that is, $\mathscr{L}$ is purely non-constant. By Theorem 3.19, $R_{u}\left(\mathrm{Gal}^{\delta}(\mathscr{U})\right)=\omega\left(\widetilde{\mathscr{L}}_{0}\right)$, where $\widetilde{\mathscr{L}}_{0}$ is the smallest $\partial$ submodule of $\mathscr{L}$ such that $\operatorname{Gal}^{\delta}\left(\mathscr{U} / \widetilde{\mathscr{L}}_{0}\right)$ is reductive. Since $\mathscr{L}$ is irreducible, either $\widetilde{\mathscr{L}}_{0}$ is zero or $\widetilde{\mathscr{L}}_{0}=\mathscr{L}$. The module $\widetilde{\mathscr{L}}_{0}$ is zero if and only if $R_{u}\left(\mathrm{Gal}^{\delta}(\mathscr{U})\right)=\{e\}$. Moreover, $R_{u}\left(\mathrm{Gal}^{\delta}(\mathscr{U})\right)=\{e\}$ if and only if $\omega(\mathscr{U})$ is a $\mathrm{Gal}^{\delta}(\mathscr{L})$-module. Since $\operatorname{dim}_{\mathrm{k}} \omega(\mathscr{L})=n$, the $\mathrm{Gal}^{\delta}(\mathscr{L})$-module $\omega(\mathscr{L})$ is not adjoint. $\operatorname{Since} \operatorname{Gal}(L)$ is a quasisimple linear algebraic group, $\operatorname{Lie}(\operatorname{Gal}(L))$ is simple (see [25, Section 14.2]), and therefore its adjoint representation is irreducible. This implies that

$$
\operatorname{Hom}_{\mathrm{Gal}(L)}(\operatorname{Lie}(\operatorname{Gal}(L)), \omega(\mathscr{L}))=0 .
$$

Therefore, by the above and Theorem 4.6, we find that $\widetilde{\mathscr{L}}_{0}$ is zero if and only if the sequence of $\mathrm{Gal}^{\delta}(\mathscr{L})$-modules

$$
0 \rightarrow \omega(\mathscr{L}) \rightarrow \omega(\mathscr{U}) \rightarrow \mathbf{k} \rightarrow 0
$$

splits, which, by [13, Theorem 3.5], is equivalent to the existence of a solution in $K$ of the equation $L(y)=b$, in contradiction with the second hypothesis. Therefore, we find that the second hypothesis is equivalent to $R_{u}(\operatorname{Gal}(\mathscr{U}))=\left(\mathbf{k}^{n},+\right)$, that is, the vector group $\mathbf{G}_{a}^{n}$ and $\mathrm{Gal}^{\delta}(\mathscr{U})=\mathbf{G}_{a}^{n} \rtimes \operatorname{Gal}(\mathscr{L})$. The latter is equivalent to $v_{1}, \ldots, v_{m}, z, \ldots, \partial^{n-1} z$ being a differential transcendence basis of $K_{\mathscr{U}}$ over $K$.

Remark 4.9 The condition in the statement of Theorem 4.7 to have no solutions $B \in K^{n \times n}$ is equivalent to the fact that $\mathrm{Gal}^{\delta}(\mathscr{L})$ is not conjugate to constants. For $K$ a computable field, this condition can be tested through various algorithms that find rational solutions (see, for instance, [3]). However, one can sometimes easily prove the non-integrability of the system by taking a close look at the topological generators of the parameterized differential Galois group such as the monodromy or the Stokes matrices. This is the strategy employed in Lemma 4.10.

\subsection{Application to the Lommel equation}

We apply Theorem 4.7 to the differential Lommel equation, which is a nonhomogeneous Bessel equation

$$
\frac{d^{2} y}{d x^{2}}+\frac{1}{x} \frac{d y}{d x}+\left(1-\frac{\alpha^{2}}{x^{2}}\right) y=x^{\mu-1},
$$

depending on two parameters, $\alpha, \mu \in \mathbb{C}$.

We will study the differential dependence of the solutions of (4.9) with respect to the parameter $\alpha$. To this purpose, we consider $\alpha$ as a new variable, transcendental over $\mathbb{C}$, and suppose that $\mu \in \mathbb{Z}$. We endow the field $\mathbb{C}(\alpha, x)$ with the derivations $\delta=\frac{\partial}{\partial \alpha}$ and $\partial=\frac{\partial}{\partial x}, \Delta=\{\delta, \partial\}$. Let $\mathbf{k}$ be a $\delta$-closure of $\mathbb{C}(\alpha)$. We extend $\partial$ to $\mathbf{k}$ as the zero derivation. We extend $\Delta$ to $K=\mathbf{k}(x)$, the field of rational functions in $x$ with coefficients 
in $\mathbf{k}$, so that $\mathbb{C}(\alpha, x)$ is a $\Delta$-subfield of $K$. Indeed, let $\mathscr{A}=\mathbf{k} \otimes_{\mathbb{C}(\alpha)} \mathbb{C}(\alpha, x)$, which is a $\Delta$-algebra over $\mathbb{C}(\alpha, x)$, and $\mathscr{A}^{\partial}=\mathbf{k}$. Since $\mathbb{C}(\alpha, x)^{\partial}=\mathbb{C}(\alpha)$, the multiplication homomorphism $\varphi: \mathscr{A} \rightarrow K$, is injective (see [29, Corollary 1, page 87]). Therefore, there is an extension of $\Delta$ onto $K$ making $\varphi$ a $\Delta$-homorphism so that $\mathbb{C}(\alpha, x) \subset K$ is a $\Delta$-field extension via $\varphi$.

Let $\mathscr{L}$ be a $\partial$-module over $K$ associated to the Bessel differential equation

$$
L(y)=\frac{d^{2} y}{d x^{2}}+\frac{1}{x} \frac{d y}{d x}+\left(1-\frac{\alpha^{2}}{x^{2}}\right) y=0
$$

and let $\mathscr{U}$ be a $\partial$-module over $K$ associated to the Lommel differential equation. We have:

$$
0 \rightarrow \mathscr{L} \rightarrow \mathscr{U} \rightarrow \mathbf{1} \rightarrow 0
$$

Lemma 4.10 The parameterized differential Galois group of $\mathscr{L}$ over $K$ is $\mathrm{SL}_{2}$.

Proof The differential Galois group of $\mathscr{L}$ over $K$ is known to be $\mathrm{SL}_{2}$ (see [28]). By [11], we know that either $\mathrm{Gal}^{\delta}(\mathscr{M})=\mathrm{SL}_{2}$ or $\mathrm{Gal}^{\delta}(\mathscr{L})$ is conjugate to constants in $\mathrm{SL}_{2}$. Suppose that we are in the second situation, that is, there exists $P \in \mathrm{SL}_{2}$ such that

$$
\operatorname{PGal}^{\delta}(\mathscr{L}) P^{-1} \subset\left\{M \in \mathrm{SL}_{2} \mid \delta(M)=0\right\} .
$$

The coefficients of (4.10) lie in $\mathbb{C}(\alpha, x)$. Moreover, for a fixed value of $\alpha$ in $\mathbb{C}$, the point zero is a parameterized regular singular point of (4.10) (see [37, Definition 2.3]). If we fix a fundamental solution $Z_{0}$ of (4.10) and follow [37, page 922], we are able to compute the parameterized monodromy matrices of (4.10) around zero. For a suitable choice of $Z_{0}$, we find the following parameterized monodromy matrix,

$$
M_{0}=\left(\begin{array}{ll}
\zeta & 0 \\
0 & \zeta
\end{array}\right)
$$

where $\zeta=e^{2 i \pi \alpha}$ and $\bar{\zeta}=e^{-2 i \pi \alpha}$ (see [38, page 35]). By [37, Theorem 3.5], $M_{0}$ belongs to some conjugate of $\mathrm{Gal}^{\delta}(\mathscr{L})$. This means that there exists $Q \in \mathrm{GL}_{2}$ such that $\delta\left(Q M_{0} Q^{-1}\right)=0$. Since conjugate matrices have the same spectrum and the spectrum of $M_{0}$ is not $\delta$-constant, we find a contradiction.

Let $J_{\alpha}(x)$ be the Bessel function of the first kind and let $Y_{\alpha}(x)$ be the Bessel function of the second kind. A solution of the Lommel differential equation is the Lommel function $s_{\mu, \alpha}(x)$, which is defined as follows

$$
s_{\mu, \alpha}(x)=\frac{1}{2} \pi\left[Y_{\alpha}(x) \int_{0}^{x} x^{\mu} J_{\alpha}(x) d x-J_{\alpha}(x) \int_{0}^{x} x^{\mu} Y_{\alpha}(x) d x\right] .
$$

Proposition 4.11 The functions, $J_{\alpha}(x), Y_{\alpha}(x), \frac{d}{d x}\left(Y_{\alpha}\right)(x), s_{\mu, \alpha}(x)$ and $\frac{d}{d x} s_{\mu, \alpha}(x)$ and all their derivatives of all order with respect to $\frac{\partial}{\partial \alpha}$ are algebraically independent over $\mathbb{C}(\alpha, x)$. Moreover, the parameterized differential Galois group of $\mathscr{U}$ is isomorphic to a semi-direct product $\mathbf{G}_{a}^{2} \rtimes \mathrm{SL}_{2}$. 
Proof $\operatorname{Since} \mathrm{Gal}^{\delta}(\mathscr{L})=\mathrm{SL}_{2}$, we just need to prove that $L(y)=x^{\mu-1}$ has no solution $g$ in $K$ in order to apply Theorem 4.7 to the Lommel differential equation. Thus, suppose on the contrary that $L(y)=x^{\mu-1}$ has a rational solution $g \in \mathbf{k}(x)$. Using partial-fraction decomposition, one can show that the only possible pole of $g$ is zero. If we write

$$
g=\sum_{j=m}^{n} a_{j} x^{j}, \quad m, n \in \mathbb{Z}, m \leq n, a_{j} \in \mathbf{k}, a_{m} a_{n} \neq 0,
$$

then the highest and lowest order terms of $L(g) \in \mathbf{k}[x, 1 / x]$ are

$$
a_{n} x^{n} \neq 0 \text { and }\left(m^{2}-\alpha^{2}\right) a_{m} x^{m-2} \neq 0,
$$

respectively. Since different powers of $x$ are linearly independent over $\mathbf{k}$ and $n \neq m-2$, $L(g)-x^{\mu-1}$ contains at least one non-zero term. Contradiction.

\section{References}

1. Arreche, C.: A Galois-theoretic proof of the differential transcendence of the incomplete Gamma function. Journal of Algebra 389, 119-127 (2013). URL http://dx.doi.org/10.1016/j.jalgebra.2013.04.037

2. Arreche, C.: Computing the differential Galois group of a parameterized second-order linear differential equation. In: Proceedings of the 39th International Symposium on Symbolic and Algebraic Computation, ISSAC 2014, pp. 43-50. ACM Press, New York (2014). URL http://dx.doi.org/10.1145/2608628.2608680

3. Barkatou, M.: A fast algorithm to compute the rational solutions of systems of linear differential equations. In: Symbolic-Numeric Analysis of Differential Equations (1997)

4. Berman, P.H., Singer, M.F.: Calculating the Galois group of $L_{1}\left(L_{2}(y)\right)=0, L_{1}, L_{2}$ completely reducible operators. Journal of Pure and Applied Algebra 139(1-3), 3-23 (1999). URL http://dx.doi.org/10.1016/S0022-4049(99)00003-1

5. Borel, A.: Properties and linear representations of Chevalley groups. In: Seminar on Algebraic Groups and Related Finite Groups, Lecture Notes in Mathematics, vol. 131, pp. 1-55. Springer (1970). URL http://dx.doi.org/10.1007/BFb0081542

6. Borel, A.: Linear Algebraic Groups, 2nd enlarged edn. Springer (1991). URL http: //dx.doi.org/10.1007/978-1-4612-0941-6

7. Bourbaki, N.: Éléments de mathématique. Livre II: Algèbre. Chapitre VIII: Modules et anneaux semisimples. Springer Berlin Heidelberg (2012)

8. Cassidy, P.: Differential algebraic groups. American Journal of Mathematics 94, 891-954 (1972). URL http://www.jstor.org/stable/2373764

9. Cassidy, P.: The differential rational representation algebra on a linear differential algebraic group. Journal of Algebra 37(2), 223-238 (1975). URL http://dx.doi.org/10.1016/0021-8693(75)90075-7

10. Cassidy, P.: Unipotent differential algebraic groups. In: Contributions to algebra: Collection of papers dedicated to Ellis Kolchin, pp. 83-115. Academic Press (1977)

11. Cassidy, P.: The classification of the semisimple differential algebraic groups and linear semisimple differential algebraic Lie algebras. Journal of Algebra 121(1), 169-238 (1989). URL http://dx.doi.org/10.1016/0021-8693(89)90092-6

12. Cassidy, P., Singer, M.: A Jordan-Hölder theorem for differential algebraic groups. Journal of Algebra 328(1), 190-217 (2011). URL http://dx.doi.org/10.1016/j.jalgebra.2010.08.019

13. Cassidy, P., Singer, M.F.: Galois theory of parametrized differential equations and linear differential algebraic group. IRMA Lectures in Mathematics and Theoretical Physics 9, 113-157 (2007). URL http://dx.doi.org/10.4171/020-1/7 
14. Deligne, P.: Catégories tannakiennes. In: The Grothendieck Festschrift, Volume II, Modern Birkhäuser Classics, pp. 111-195. Birkhäuser, Boston, MA (1990). URL http://dx.doi .org/10.1007/978-0-8176-4575-5

15. Demazure, M., Gabriel, P.: Groupes algébriques. Tome I: Géométrie algébrique, généralités, groupes commutatifs. Masson \& Cie, Éditeur, Paris (1970). Avec un appendice it Corps de classes local par Michiel Hazewinkel

16. Di Vizio, L., Hardouin, C., Wibmer, M.: Difference algebraic relations among solutions of linear differential equations. Journal of the Institute of Mathematics of Jussieu (2015). URL http://dx.doi.org/10.1017/S1474748015000080

17. Dreyfus, T.: Computing the Galois group of some parameterized linear differential equation of order two. Proceedings of the American Mathematical Society 142, 1193-1207 (2014). URL http://dx.doi.org/10.1090/S0002-9939-2014-11826-0

18. Dreyfus, T., Hardouin, C., Roques, J.: Hypertranscendance of solutions of Mahler equations (2015). URL http: //arxiv.org/abs/1507.03361. To appear in the Journal of the European Mathematical Society

19. Feng, R.: Hrushovski's algorithm for computing the Galois group of a linear differential equation. Advances in Applied Mathematics 65, 1-37 (2015). URL http://dx.doi.org/10.1016/j.aam.2015.01.001

20. Gillet, H., Gorchinskiy, S., Ovchinnikov, A.: Parameterized Picard-Vessiot extensions and Atiyah extensions. Advances in Mathematics 238, 322-411 (2013). URL http://dx.doi.org/10.1016/j.aim.2013.02.006

21. Hardouin, C.: Unipotent radicals of Tannakian Galois groups in positive characteristic. In: Arithmetic and Galois theories of differential equations, Sémin. Congr., vol. 23, pp. 223-239. Soc. Math. France, Paris (2011)

22. Hardouin, C., Singer, M.F.: Differential Galois theory of linear difference equations. Mathematische Annalen 342(2), 333-377 (2008). URL http: //dx . doi .org/10.1007/s00208-008-0238-z

23. van Hoeij, M.: Factorization of differential operators with rational functions coefficients. Journal of Symbolic Computation 24(5), 537-561 (1997). URL http://dx. doi .org/10.1006/jsco.1997.0151

24. Hrushovski, E.: Computing the Galois group of a linear differential equation. In: Differential Galois theory (Bedlewo, 2001), Banach Center Publ., vol. 58, pp. 97-138. Polish Acad. Sci., Warsaw (2002). URL http://dx.doi.org/10.4064/bc58-0-9

25. Humphreys, J.E.: Linear algebraic groups. Springer-Verlag, New York (1975). URL http://dx.doi.org/10.1007/978-1-4684-9443-3

26. Kamensky, M.: Model theory and the Tannakian formalism. Transactions of the American Mathematical Society 367, 1095-1120 (2015). URL http: //dx . doi .org/10.1090/S0002-9947-2014-06062-5

27. Kaplansky, I.: An introduction to differential algebra. Hermann, Paris (1957)

28. Kolchin, E.: Algebraic groups and algebraic dependence. American Journal of Mathematics 90(4), 11511164 (1968). URL http://dx.doi .org/10 . 2307/2373294

29. Kolchin, E.: Differential Algebra and Algebraic Groups. Academic Press, New York (1973)

30. Kurkova, I., Raschel, K.: On the functions counting walks with small steps in the quarter plane. Publications Mathématiques. Institut des Hautes Études Scientifiques 116(1), 69-114 (2012). URL http://dx.doi.org/10.1007/s10240-012-0045-7

31. Magid, A.: Lectures on Differential Galois Theory. American Mathematical Society, Providence, RI (1994)

32. Marker, D.: Model theory of differential fields. In: Model theory, algebra, and geometry, Mathematical Sciences Research Institute Publications, vol. 39, pp. 53-63. Cambridge University Press, Cambridge (2000). URL http://library.msri.org/books/Book39/files/dcf .pdf

33. Minchenko, A., Ovchinnikov, A.: Zariski closures of reductive linear differential algebraic groups. Advances in Mathematics 227(3), 1195-1224 (2011). URL http://dx.doi.org/10.1016/j.aim.2011.03.002

34. Minchenko, A., Ovchinnikov, A.: Extensions of differential representations of $\mathrm{SL}_{2}$ and tori. Journal of the Institute of Mathematics of Jussieu 12(1), 199-224 (2013). URL http://dx.doi.org/10.1017/S1474748012000692

35. Minchenko, A., Ovchinnikov, A., Singer, M.F.: Unipotent differential algebraic groups as parameterized differential Galois groups. Journal of the Institute of Mathematics of Jussieu 13(4), 671-700 (2014). URL http://dx.doi.org/10.1017/S1474748013000200 
36. Minchenko, A., Ovchinnikov, A., Singer, M.F.: Reductive linear differential algebraic group and the Galois groups of parametrized linear differential equations. International Mathematics Research Notices 2015(7), 1733-1793 (2015). URL http://dx.doi.org/10.1093/imrn/rnt344

37. Mitschi, C., Singer, M.F.: Monodromy groups of parameterized linear differential equations with regular singularities. Bulletin of the London Mathematical Society 44(5), 913-930 (2012). URL http://dx.doi.org/10.1112/blms/bds021

38. Morales Ruiz, J.J.: Differential Galois theory and non-integrability of Hamiltonian systems. Modern Birkhäuser Classics. Birkhäuser/Springer, Basel (1999). URL http://dx.doi.org/10.1007/978-3-0348-8718-2

39. Nagloo, J., León Sánchez, O.: On parameterized differential Galois extensions. Journal of Pure and Applied Algebra 220(7), 2549-2563 (2016). URL http://dx.doi.org/10.1016/j.jpaa.2015.12.001

40. Nguyen, P.: Hypertranscedance de fonctions de Mahler du premier ordre. C. R. Math. Acad. Sci. Paris 349(17-18), 943-946 (2011). URL http://dx.doi.org/10.1016/j.crma.2011.08.021

41. Nishioka, K.: A note on differentially algebraic solutions of first order linear difference equations. Aequationes Mathematicae 27(1-2), 32-48 (1984). URL http://dx.doi .org/10 .1007/BF02192657

42. Ostrowski, A.: Sur les relations algébriques entre les intégrales indéfinies. Acta Mathematica 78, 315318 (1946). URL http://dx.doi.org/10.1007/BF02421605

43. Ovchinnikov, A.: Tannakian approach to linear differential algebraic groups. Transformation Groups 13(2), 413-446 (2008). URL http://dx.doi .org/10.1007/s00031-008-9010-4

44. Ovchinnikov, A.: Tannakian categories, linear differential algebraic groups, and parametrized linear differential equations. Transformation Groups 14(1), 195-223 (2009). URL http://dx.doi.org/10.1007/s00031-008-9042-9

45. van der Put, M., Singer, M.F.: Galois theory of linear differential equations. Springer-Verlag, Berlin (2003). URL http://dx.doi .org/10.1007/978-3-642-55750-7

46. Randé, B.: Équations fonctionnelles de Mahler et applications aux suites p-régulières. Ph.D. thesis, Université Bordeaux I (1992). URL https://tel.archives-ouvertes .fr/tel-01183330

47. Ritt, J.F.: Differential Algebra. American Mathematical Society Colloquium Publications, Vol. XXXIII. American Mathematical Society, New York, N. Y. (1950)

48. Singer, M.F.: Testing reducibility of linear differential operators: a group-theoretic perspective. Applicable Algebra in Engineering, Communication and Computing 7(2), 77-104 (1996). URL http://dx.doi.org/10.1007/BF01191378

49. Singer, M.F.: Linear algebraic groups as parameterized Picard-Vessiot Galois groups. Journal of Algebra 373, 153-161 (2013). URL http://dx.doi.org/10.1016/j·jalgebra.2012.09.037

50. Springer, T.A.: Invariant theory. Springer-Verlag, Berlin-New York (1977).

URL http://dx.doi.org/10.1007/BFb0095644

51. Vinberg, E.B.: A Course in Algebra. American Mathematical Society, Providence, RI (2003). URL http://dx.doi.org/10.1090/gsm/056

52. Waterhouse, W.C.: Introduction to affine group schemes, Graduate Texts in Mathematics, vol. 66. Springer-Verlag, New York (1979). URL http://dx.doi.org/10.1007/978-1-4612-6217-6

53. Wibmer, M.: Existence of $\partial$-parameterized Picard-Vessiot extensions over fields with algebraically closed constants. Journal of Algebra 361, 163-171 (2012). URL http://dx.doi.org/10.1016/j.jalgebra.2012.03.035 